\title{
Bifurcation Analysis for an SEIRS-V Model with Delays on the Transmission of Worms in a Wireless Sensor Network
}

\author{
Zizhen Zhang and Yougang Wang \\ School of Management Science and Engineering, Anhui University of Finance and Economics, Bengbu 233030, China \\ Correspondence should be addressed to Zizhen Zhang; zzzhaida@163.com
}

Received 10 February 2016; Accepted 23 May 2016; Published 18 January 2017

Academic Editor: Vladimir Turetsky

Copyright (C) 2017 Z. Zhang and Y. Wang. This is an open access article distributed under the Creative Commons Attribution License, which permits unrestricted use, distribution, and reproduction in any medium, provided the original work is properly cited.

Hopf bifurcation for an SEIRS-V model with delays on the transmission of worms in a wireless sensor network is investigated. We focus on existence of the Hopf bifurcation by regarding the diverse delay as a bifurcation parameter. The results show that propagation of worms in the wireless sensor network can be controlled when the delay is suitably small under some certain conditions. Then, we study properties of the Hopf bifurcation by using the normal form theory and center manifold theorem. Finally, we give a numerical example to support the theoretical results.

\section{Introduction}

In recent years, wireless sensor networks have received extensive attention due to their vast potential in many application environments. However, security of wireless sensor networks still remains one of the most critical challenges because sensor nodes are often placed in a hostile or dangerous environment [1]. Many epidemiological models [2-6] have been proposed to study and predict the spread of viruses in wireless networks motivated by the pioneering work of Murray [7] and Kephart and White [8, 9]. In [10], Mishra and Keshri proposed the following SEIRS-V model to describe the propagation of worms in a wireless sensor network:

$$
\begin{aligned}
\frac{d S(t)}{d t}= & A-\beta S(t) I(t)-(\zeta+p) S(t)+\delta R(t) \\
& +\eta V(t), \\
\frac{d E(t)}{d t}= & \beta S(t) I(t)-(\zeta+\alpha) E(t), \\
\frac{d I(t)}{d t}= & \alpha E(t)-(\zeta+\varepsilon+\gamma) I(t),
\end{aligned}
$$

$$
\begin{aligned}
& \frac{d R(t)}{d t}=\gamma I(t)-(\zeta+\delta) R(t), \\
& \frac{d V(t)}{d t}=p S(t)-(\zeta+\eta) V(t),
\end{aligned}
$$

where $S(t), E(t), I(t), R(t)$, and $V(t)$ denote the number of susceptible, exposed (infected, but not infectious), infectious, recovered, and vaccinated sensor nodes at time $t$, respectively. $A, p, \alpha, \beta, \gamma, \delta, \eta, \varepsilon$, and $\zeta$ are the positive parameters of system (1) and for the specific meanings of them one can refer to [10]. Considering the time delays in system (1), Zhang and Si [11] proposed the following delayed SEIRS-V system:

$$
\begin{aligned}
\frac{d S(t)}{d t}= & A-\beta S(t) I(t)-(\zeta+p) S(t)+\delta R\left(t-\tau_{2}\right) \\
& +\eta V\left(t-\tau_{2}\right), \\
\frac{d E(t)}{d t}= & \beta S(t) I(t)-(\zeta+\alpha) E(t),
\end{aligned}
$$




$$
\begin{aligned}
& \frac{d I(t)}{d t}=\alpha E(t)-(\zeta+\varepsilon) I(t)-\gamma I\left(t-\tau_{1}\right), \\
& \frac{d R(t)}{d t}=\gamma I\left(t-\tau_{1}\right)-\zeta R(t)-\delta R\left(t-\tau_{2}\right), \\
& \frac{d V(t)}{d t}=p S(t)-\zeta V(t)-\eta \mathrm{V}\left(t-\tau_{2}\right),
\end{aligned}
$$

where $\tau_{1} \geq 0$ is the time delay due to the period that antivirus software uses to clean worms in the infected nodes; $\tau_{2} \geq 0$ is the time delay due to the temporary immunity period of the recovered and the vaccinated nodes. For the convenience of analysis, Zhang and $\mathrm{Si}$ [11] let $\tau_{1}=\tau_{2}$ and considered the following system:

$$
\begin{aligned}
\frac{d S(t)}{d t}= & A-\beta S(t) I(t)-(\zeta+p) S(t)+\delta R(t-\tau) \\
& +\eta V(t-\tau), \\
\frac{d E(t)}{d t}= & \beta S(t) I(t)-(\zeta+\alpha) E(t), \\
\frac{d I(t)}{d t}= & \alpha E(t)-(\zeta+\varepsilon) I(t)-\gamma I(t-\tau), \\
\frac{d R(t)}{d t}= & \gamma I(t-\tau)-\zeta R(t)-\delta R(t-\tau), \\
\frac{d V(t)}{d t}= & p S(t)-\zeta V(t)-\eta V(t-\tau) .
\end{aligned}
$$

Zhang and Si [11] investigated existence and properties of the Hopf bifurcation of system (3).

It should be pointed out that one of the significant features of worms in networks is its latent characteristic. Therefore, there exists a certain period before the exposed nodes develop themselves into the infectious ones. In addition, as far as we know, there have been some papers that deal with research of Hopf bifurcation of dynamical systems with multiple delays in recent years [12-15]. In [12], Xu et al. studied Hopf bifurcation of a ring of five neurons with delays. In [14], Bianca et al. investigated Hopf bifurcation of an economic growth model with two delays. Considering that there is a latent period of worms in the exposed nodes in system (3), we study the following system with delays:

$$
\begin{aligned}
\frac{d S(t)}{d t}= & A-\beta S(t) I(t)-(\zeta+p) S(t)+\delta R\left(t-\tau_{2}\right) \\
& +\eta V\left(t-\tau_{2}\right), \\
\frac{d E(t)}{d t}= & \beta S(t) I(t)-\alpha E\left(t-\tau_{1}\right)-\zeta E(t), \\
\frac{d I(t)}{d t}= & \alpha E\left(t-\tau_{1}\right)-(\zeta+\varepsilon) I(t)-\gamma I\left(t-\tau_{2}\right), \\
\frac{d R(t)}{d t}= & \gamma I\left(t-\tau_{2}\right)-\zeta R(t)-\delta R\left(t-\tau_{2}\right), \\
\frac{d V(t)}{d t}= & p S(t)-\zeta V(t)-\eta V\left(t-\tau_{2}\right),
\end{aligned}
$$

where $\tau_{1}$ is the time delay due to the latent period of worms in the exposed nodes and $\tau_{2}$ is the time delay due to the period that the antivirus software uses to clean worms in the infected nodes and that due to the temporary immunity period of the recovered and the vaccinated nodes.

The structure of this paper is as follows. In Section 2, we obtain sufficient conditions for local stability of the positive equilibrium and existence of a Hopf bifurcation of system (4). In Section 3, we deal with the properties of the Hopf bifurcation by using the normal form theory and center manifold theorem. Some numerical simulations are carried out in Section 4 with the aim of verifying the obtained analytic results. Finally, conclusions and future work are summarized.

\section{Hopf Bifurcation Analysis}

By a direct computation, we know that if $R_{0}=(\alpha \beta A(\zeta+$ $\eta)+p \eta(\zeta+\alpha)(\zeta+\varepsilon+\gamma)) /(\zeta+p)(\zeta+\alpha)(\zeta+\eta)(\zeta+\varepsilon+$ $\gamma)>1$, then system (4) has a unique positive equilibrium $D_{*}\left(S_{*}, E_{*}, I_{*}, R_{*}, V_{*}\right)$ in which

$$
\begin{aligned}
S_{*} & =\frac{(\zeta+\alpha)(\zeta+\varepsilon+\gamma)}{\alpha \beta}, \\
E_{*} & =\frac{\zeta+\varepsilon+\gamma}{\alpha} I_{*}, \\
R_{*} & =\frac{\gamma}{\zeta+\delta} I_{*}, \\
V_{*} & =\frac{p(\zeta+\alpha)(\zeta+\varepsilon+\gamma)}{\alpha \beta(\zeta+\eta)}, \\
I_{*} & =\frac{\alpha \beta A(\zeta+\delta)(\zeta+\eta)+p \eta(\zeta+\alpha)(\zeta+\delta)(\zeta+\varepsilon+\gamma)-(\zeta+p)(\zeta+\alpha)(\zeta+\delta)(\zeta+\eta)(\zeta+\varepsilon+\gamma)}{\beta(\zeta+\alpha)(\zeta+\delta)(\zeta+\eta)(\zeta+\varepsilon+\gamma)-\alpha \beta \delta \gamma(\zeta+\eta)} .
\end{aligned}
$$


The characteristic equation of system (4) at $D_{*}$ is

$$
\begin{aligned}
\lambda^{5}+ & A_{4} \lambda^{4}+A_{3} \lambda^{3}+A_{2} \lambda^{2}+A_{1} \lambda+A_{0} \\
& +\left(B_{4} \lambda^{4}+B_{3} \lambda^{3}+B_{2} \lambda^{2}+B_{1} \lambda+B_{0}\right) e^{-\lambda \tau_{1}} \\
& +\left(C_{4} \lambda^{4}+C_{3} \lambda^{3}+C_{2} \lambda^{2}+C_{1} \lambda+C_{0}\right) e^{-\lambda \tau_{2}} \\
& +\left(D_{3} \lambda^{3}+D_{2} \lambda^{2}+D_{1} \lambda+D_{0}\right) e^{-2 \lambda \tau_{2}} \\
& +\left(E_{3} \lambda^{3}+E_{2} \lambda^{2}+E_{1} \lambda+E_{0}\right) e^{-\lambda\left(\tau_{1}+\tau_{2}\right)} \\
& +\left(F_{2} \lambda^{2}+F_{1} \lambda+F_{0}\right) e^{-\lambda\left(\tau_{1}+2 \tau_{2}\right)} \\
& +\left(G_{2} \lambda^{2}+G_{1} \lambda+G_{0}\right) e^{-3 \lambda \tau_{2}} \\
& +\left(H_{1} \lambda+H_{0}\right) e^{-\lambda\left(\tau_{1}+3 \tau_{2}\right)}=0,
\end{aligned}
$$

where

$$
\begin{aligned}
A_{0}= & -a_{11} a_{22} a_{33} a_{44} a_{55}, \\
A_{1}= & a_{11} a_{22} a_{33} a_{44}+a_{11} a_{22} a_{55}\left(a_{33}+a_{44}\right) \\
& +a_{33} a_{44} a_{55}\left(a_{11}+a_{22}\right), \\
A_{2}= & -a_{55}\left(a_{11} a_{22}+a_{33} a_{44}+\left(a_{11}+a_{22}\right)\left(a_{33}+a_{44}\right)\right) \\
& -\left(a_{11} a_{22}\left(a_{33}+a_{44}\right)+a_{33} a_{44}\left(a_{11}+a_{22}\right)\right), \\
A_{3}= & a_{11} a_{22}+a_{33} a_{44}+\left(a_{11}+a_{22}\right)\left(a_{33}+a_{44}\right) \\
& +a_{55}\left(a_{11}+a_{22}+a_{33}+a_{44}\right), \\
A_{4}= & -\left(a_{11}+a_{22}+a_{33}+a_{44}+a_{55}\right), \\
B_{0}= & a_{44} a_{55} b_{32}\left(a_{11} a_{23}-a_{13} a_{21}\right)-a_{11} a_{22} a_{33} a_{44} b_{22}, \\
B_{1}= & b_{22}\left(a_{11} a_{22}\left(a_{33}+a_{44}\right)+a_{33} a_{44}\left(a_{11}+a_{22}\right)\right) \\
& +b_{32}\left(\left(a_{44}+a_{55}\right)\left(a_{13} a_{21}-a_{23} a_{11}\right)-a_{23} a_{44} a_{55}\right), \\
B_{2}= & a_{23} b_{32}\left(a_{44}+a_{55}\right)+b_{32}\left(a_{11} a_{23}-a_{13} a_{21}\right) \\
& -b_{22}\left(a_{11} a_{22}+a_{33} a_{44}+\left(a_{11}+a_{22}\right)\left(a_{33}+a_{44}\right)\right), \\
B_{3}= & b_{22}\left(a_{11}+a_{22}+a_{33}+a_{44}\right)-a_{23} b_{32}, \\
B_{4}= & -b_{22}, \\
C_{0}= & -a_{11} a_{22} a_{33}\left(a_{44} b_{55}+a_{55} b_{44}\right)-a_{11} a_{22} a_{44} a_{55} b_{33}, \\
C_{1}= & b_{33}\left(a_{11} a_{22}\left(a_{44}+a_{55}\right)+a_{44} a_{55}\left(a_{11}+a_{22}\right)\right) \\
& +\left(a_{11} a_{22}+a_{11} a_{33}+a_{22} a_{33}\right)\left(a_{44} b_{55}+a_{55} b_{44}\right) \\
& +a_{11} a_{22} a_{33}\left(b_{44}+b_{55}\right), \\
C_{2}= & -\left(a_{11} a_{22}+a_{11} a_{33}+a_{22} a_{33}\right)\left(b_{44}+b_{55}\right) \\
& -\left(a_{44} b_{55}+a_{55} b_{44}\right)\left(a_{11}+a_{22}+a_{33}\right) \\
& \left.a_{22}+a_{44} a_{55}+\left(a_{11}+a_{22}\right)\left(a_{44}+a_{55}\right)\right),
\end{aligned}
$$

$$
\begin{aligned}
C_{3}= & b_{33}\left(a_{11}+a_{22}+a_{44}+a_{55}\right)+a_{44} b_{55}+a_{55} b_{44} \\
& +\left(a_{11}+a_{22}+a_{33}\right)\left(b_{44}+b_{55}\right), \\
C_{4}= & -\left(b_{22}+b_{44}+b_{55}\right), \\
D_{0}= & -a_{11} a_{22}\left(a_{33} b_{44} b_{55}+a_{44} b_{33} b_{55}+a_{55} b_{33} b_{44}\right), \\
D_{1}= & b_{33} b_{44}\left(a_{11} a_{22}+a_{11} a_{55}+a_{22} a_{55}\right) \\
& +b_{33} b_{55}\left(a_{11} a_{22}+a_{11} a_{44}+a_{22} a_{44}\right) \\
& +b_{44} b_{55}\left(a_{11} a_{22}+a_{11} a_{33}+a_{22} a_{33}\right), \\
D_{2}= & -b_{33} b_{44}\left(a_{11}+a_{22}+a_{55}\right) \\
& -b_{33} b_{55}\left(a_{11}+a_{22}+a_{44}\right) \\
& -b_{44} b_{55}\left(a_{11}+a_{22}+a_{33}\right),
\end{aligned}
$$$$
D_{3}=b_{33} b_{44}+b_{33} b_{55}+b_{44} b_{55} \text {, }
$$$$
E_{0}=b_{32}\left(a_{44} b_{55}+a_{55} b_{44}\right)\left(a_{11} a_{23}-a_{13} a_{21}\right)
$$$$
-a_{11} b_{22}\left(a_{44} a_{55} b_{33}+a_{33} a_{44} b_{55}+a_{33} a_{55} b_{44}\right),
$$$$
E_{1}=a_{11} a_{33} b_{22}\left(b_{44}+b_{55}\right)
$$$$
+b_{22} b_{33}\left(a_{11} a_{44}+a_{11} a_{55}+a_{44} a_{55}\right)
$$$$
+\left(a_{11} b_{22}+a_{33} b_{22}-a_{23} b_{32}\right)\left(a_{44} b_{55}+a_{55} b_{44}\right)
$$$$
+b_{32}\left(b_{44}+b_{55}\right)\left(a_{13} a_{21}-a_{11} a_{23}\right) \text {, }
$$$$
E_{2}=a_{23} b_{32}\left(b_{44}+b_{55}\right)-b_{22} b_{33}\left(a_{11}+a_{44}+a_{55}\right)
$$$$
-b_{22}\left(a_{44} b_{55}+a_{55} b_{44}+\left(b_{44}+b_{55}\right)\left(a_{11}+a_{33}\right)\right) \text {, }
$$$$
E_{3}=b_{22}\left(b_{33}+b_{44}+b_{55}\right) \text {, }
$$$$
F_{0}=a_{21} a_{55} b_{14} b_{32} b_{43}+b_{32} b_{44} b_{55}\left(a_{11} a_{23}-a_{13} a_{21}\right)
$$$$
-a_{11} b_{22}\left(a_{33} b_{44} b_{55}+a_{44} b_{33} b_{55}+a_{55} b_{33} b_{44}\right) \text {, }
$$$$
F_{1}=b_{22} b_{33} b_{55}\left(a_{11}+a_{44}\right)+b_{22} b_{44} b_{55}\left(a_{11}+a_{33}\right)
$$$$
-\left(a_{21} b_{14} b_{32} b_{43}+a_{23} a_{32} b_{44} b_{55}\right),
$$$$
F_{2}=-b_{22}\left(b_{33} b_{44}+b_{33} b_{55}+b_{44} b_{55}\right) \text {, }
$$$$
G_{0}=-a_{11} a_{22} b_{33} b_{44} b_{55} \text {, }
$$$$
G_{1}=b_{33} b_{44} b_{55}\left(a_{11}+a_{22}\right) \text {, }
$$$$
G_{2}=-b_{33} b_{44} b_{55} \text {, }
$$$$
H_{0}=a_{21} b_{14} b_{32} b_{43} b_{55}-a_{11} b_{22} b_{33} b_{44} b_{55} \text {, }
$$$$
H_{1}=b_{22} b_{33} b_{44} b_{55} \text {, }
$$ 
with

$$
\begin{aligned}
& a_{11}=-\left(\beta I_{*}+\zeta+p\right), \\
& a_{13}=-\beta S_{*}, \\
& a_{21}=\beta I_{*}, \\
& a_{22}=-\zeta, \\
& a_{23}=\beta S_{*}, \\
& a_{33}=-(\zeta+\varepsilon), \\
& a_{44}=-\zeta, \\
& a_{51}=p, \\
& a_{55}=-\zeta, \\
& b_{14}=\delta, \\
& b_{15}=\eta, \\
& b_{22}=-\alpha, \\
& b_{32}=\alpha, \\
& b_{33}=-\gamma, \\
& b_{43}=\gamma, \\
& b_{44}=-\delta, \\
& b_{55}=-\eta .
\end{aligned}
$$

Case 1. When $\tau_{1}=\tau_{2}=0$, (6) becomes

$$
\lambda^{5}+A_{14} \lambda^{4}+A_{13} \lambda^{3}+A_{12} \lambda^{2}+A_{11} \lambda+A_{10}=0,
$$

where

$$
\begin{aligned}
& A_{10}=A_{0}+B_{0}+C_{0}+D_{0}+E_{0}+F_{0}+G_{0}+H_{0}, \\
& A_{11}=A_{1}+B_{1}+C_{1}+D_{1}+E_{1}+F_{1}+G_{1}+H_{1}, \\
& A_{12}=A_{2}+B_{2}+C_{2}+D_{2}+E_{2}+F_{2}+G_{2}, \\
& A_{13}=A_{3}+B_{3}+C_{3}+D_{3}+E_{3}, \\
& A_{14}=A_{4}+B_{4}+C_{4} .
\end{aligned}
$$

It is easy to get that $A_{14}=p+\alpha+\gamma+\delta+\varepsilon+\eta+5 \zeta+\beta I_{*}>0$. Thus, we have the following condition:

$\left(H_{1}\right)$ If (11) holds, $D_{*}$ is locally asymptotically stable:

$$
\operatorname{det}_{2}=\left(\begin{array}{cc}
A_{14} & 1 \\
A_{12} & A_{13}
\end{array}\right)>0
$$

$$
\begin{aligned}
\operatorname{det}_{3} & =\left(\begin{array}{ccc}
A_{14} & 1 & 0 \\
A_{12} & A_{13} & A_{14} \\
0 & A_{11} & A_{12}
\end{array}\right)>0, \\
\operatorname{det}_{4} & =\left(\begin{array}{cccc}
A_{14} & 1 & 0 & 0 \\
A_{12} & A_{13} & A_{14} & 1 \\
A_{10} & A_{11} & A_{12} & A_{13} \\
0 & 0 & A_{10} & A_{11}
\end{array}\right)>0, \\
\operatorname{det}_{5} & =\left(\begin{array}{ccccc}
A_{14} & 1 & 0 & 0 & 0 \\
A_{12} & A_{13} & A_{14} & 1 & 0 \\
A_{10} & A_{11} & A_{12} & A_{13} & A_{14} \\
0 & 0 & A_{10} & A_{11} & A_{12} \\
0 & 0 & 0 & 0 & A_{10}
\end{array}\right)>0 .
\end{aligned}
$$

Case 2. When $\tau_{1}>0, \tau_{2}=0$, (6) becomes

$$
\begin{aligned}
\lambda^{5}+ & A_{24} \lambda^{4}+A_{23} \lambda^{3}+A_{22} \lambda^{2}+A_{21} \lambda+A_{20} \\
& +\left(B_{24} \lambda^{4}+B_{23} \lambda^{3}+B_{22} \lambda^{2}+B_{21} \lambda+B_{20}\right) e^{-\lambda \tau_{1}} \\
= & 0,
\end{aligned}
$$

where

$$
\begin{aligned}
& A_{20}=A_{0}+C_{0}+D_{0}+G_{0}, \\
& A_{21}=A_{1}+C_{1}+D_{1}+G_{1}, \\
& A_{22}=A_{2}+C_{2}+D_{2}+G_{2}, \\
& A_{23}=A_{3}+C_{3}+D_{3}, \\
& A_{24}=A_{4}+C_{4}, \\
& B_{20}=B_{0}+E_{0}+F_{0}+H_{0}, \\
& B_{21}=B_{1}+E_{1}+F_{1}+H_{1}, \\
& B_{22}=B_{2}+E_{2}+F_{2}, \\
& B_{23}=B_{3}+E_{3}, \\
& B_{24}=B_{4} .
\end{aligned}
$$

Let $\lambda=i \omega_{1}\left(\omega_{1}>0\right)$ be a root of (12). Then,

$$
\begin{aligned}
& \left(B_{21} \omega_{1}-B_{23} \omega_{1}^{3}\right) \sin \tau_{1} \omega_{1} \\
& \quad+\left(B_{24} \omega_{1}^{4}-B_{22} \omega_{1}^{2}+B_{20}\right) \cos \tau_{1} \omega_{1}=A_{22} \omega_{1}^{2} \\
& \quad-A_{24} \omega_{1}^{4}-A_{20}, \\
& \left(B_{21} \omega_{1}-B_{23} \omega_{1}^{3}\right) \cos \tau_{1} \omega_{1} \\
& \quad-\left(B_{24} \omega_{1}^{4}-B_{22} \omega_{1}^{2}+B_{20}\right) \sin \tau_{1} \omega_{1}=A_{23} \omega_{1}^{3}-\omega_{1}^{5} \\
& \quad-A_{21} \omega_{1} .
\end{aligned}
$$


Thus, we have

$$
\omega_{1}^{10}+s_{24} \omega_{1}^{8}+s_{23} \omega_{1}^{6}+s_{22} \omega_{1}^{4}+s_{21} \omega_{1}+s_{20}=0,
$$

where

$$
\begin{aligned}
s_{20}= & A_{20}^{2}-B_{20}^{2}, \\
s_{21}= & A_{21}^{2}-B_{21}^{2}-2 A_{20} A_{22}+2 B_{20} B_{22}, \\
s_{22}= & A_{22}^{2}-B_{22}^{2}+2 A_{20} A_{24}-2 A_{21} A_{23}-2 B_{20} B_{24} \\
& +2 B_{21} B_{23}, \\
s_{23}= & A_{23}^{2}-B_{23}^{2}+2 A_{21}-2 A_{22} A_{24}+2 B_{22} B_{24}, \\
s_{24}= & A_{24}^{2}-B_{24}^{2}-2 A_{23} .
\end{aligned}
$$

Let $\omega_{1}^{2}=v_{1}$; then, (15) becomes

$$
v_{1}^{5}+s_{24} v_{1}^{4}+s_{23} v_{1}^{3}+s_{22} v_{1}^{2}+s_{21} v_{1}+s_{20}=0 .
$$

According to the analysis of roots of (17) in [16], we assume that

$\left(H_{21}\right)$ Equation (17) has at least one positive root.

Then, there exists a positive root $v_{10}$ for (17). Thus, we obtain $\omega_{10}=\sqrt{v_{10}}$. Further,

$$
\begin{aligned}
\tau_{10} & =\frac{1}{\omega_{10}} \\
& \times \arccos \frac{p_{28} \omega_{10}^{8}+p_{26} \omega_{10}^{6}+p_{24} \omega_{10}^{4}+p_{22} \omega_{10}^{2}+p_{20}}{q_{28} \omega_{10}^{8}+q_{26} \omega_{10}^{6}+q_{284} \omega_{10}^{4}+q_{22} \omega_{10}^{2}+q_{20}},
\end{aligned}
$$

where

$$
\begin{aligned}
& q_{20}=B_{20}^{2}, \\
& q_{22}=B_{21}^{2}-2 B_{22}, \\
& q_{24}=B_{22}^{2}+2 B_{20} B_{24}-B_{21} B_{23}, \\
& q_{26}=B_{23}^{2}-2 B_{22} B_{24}, \\
& q_{28}=B_{24}^{2}, \\
& p_{20}=-A_{20} B_{20}, \\
& p_{22}=A_{20} B_{22}+A_{22} B_{20}-A_{21} B_{21}, \\
& p_{24}=A_{21} B_{23}-A_{20} B_{24}-A_{22} B_{22}+A_{23} B_{21}-A_{24} B_{20}, \\
& p_{26}=A_{22} B_{24}+A_{24} B_{22}-A_{23} B_{23}-B_{21}, \\
& p_{28}=B_{23}-A_{24} B_{24} .
\end{aligned}
$$

Differentiating (12) regarding $\tau_{1}$, we have

$$
\begin{aligned}
& {\left[\frac{d \lambda}{d \tau_{1}}\right]^{-1}} \\
& =-\frac{5 \lambda^{4}+4 A_{24} \lambda^{3}+3 A_{23} \lambda^{2}+2 A_{22} \lambda+A_{21}}{\lambda\left(\lambda^{5}+A_{24} \lambda^{4}+A_{23} \lambda^{3}+A_{22} \lambda^{2}+A_{21} \lambda+A_{20}\right)} \\
& \quad+\frac{4 B_{24} \lambda^{3}+3 B_{23} \lambda^{2}+2 B_{22} \lambda+B_{21}}{\lambda\left(B_{24} \lambda^{4}+B_{23} \lambda^{3}+B_{22} \lambda^{2}+B_{21} \lambda+B_{20}\right)}-\frac{\tau_{1}}{\lambda} .
\end{aligned}
$$

Then,

$$
\begin{aligned}
& \operatorname{Re}\left[\frac{d \lambda}{d \tau_{1}}\right]_{\lambda=i \omega_{10}}^{-1} \\
& =\frac{f_{21}^{\prime}\left(v_{1 *}\right)}{\left(B_{21} \omega_{10}-B_{23} \omega_{10}^{3}\right)^{2}+\left(B_{24} \omega_{10}^{4}-B_{22} \omega_{10}^{2}+B_{20}\right)^{2}}
\end{aligned}
$$

where

$$
f_{21}\left(v_{1}\right)=v_{1}^{5}+s_{24} v_{1}^{4}+s_{23} v_{1}^{3}+s_{22} v_{1}^{2}+s_{21} v_{1}+s_{20}
$$

and $v_{1 *}=\omega_{10}^{2}$.

Thus, we can conclude that if

$$
\left(H_{22}\right) f_{21}^{\prime}\left(v_{1 *}\right) \neq 0 \text { holds, then } \operatorname{Re}\left[d \lambda / d \tau_{1}\right]_{\lambda=i \omega_{10}}^{-1} \neq 0 \text {. }
$$

Summarizing the analysis above, we have the following.

Theorem 1. For system (4), let $\tau_{10}$ be specified by (18). If the conditions $\left(H_{21}\right)-\left(H_{22}\right)$ are satisfied, then $D_{*}\left(S_{*}, E_{*}, I_{*}\right.$, $\left.R_{*}, V_{*}\right)$ is asymptotically stable when $\tau_{1} \in\left[0, \tau_{10}\right)$ and a Hopf bifurcation occurs at $D_{*}\left(S_{*}, E_{*}, I_{*}, R_{*}, V_{*}\right)$ when $\tau_{1}=\tau_{10}$.

Case 3. When $\tau_{1}=0$ and $\tau_{2}>0$, (6) becomes

$$
\begin{aligned}
\lambda^{5}+ & A_{34} \lambda^{4}+A_{33} \lambda^{3}+A_{32} \lambda^{2}+A_{31} \lambda+A_{30} \\
& +\left(B_{34} \lambda^{4}+B_{33} \lambda^{3}+B_{32} \lambda^{2}+B_{31} \lambda+B_{30}\right) e^{-\lambda \tau_{2}} \\
& +\left(C_{33} \lambda^{3}+C_{32} \lambda^{2}+C_{31} \lambda+C_{30}\right) e^{-2 \lambda \tau_{2}} \\
& +\left(D_{32} \lambda^{2}+D_{31} \lambda+D_{30}\right) e^{-3 \lambda \tau_{2}}=0 .
\end{aligned}
$$

Multiplying by $e^{\lambda \tau_{2}}$ on both sides of (23), we have

$$
\begin{aligned}
& B_{34} \lambda^{4}+B_{33} \lambda^{3}+B_{32} \lambda^{2}+B_{31} \lambda+B_{30} \\
& +\left(\lambda^{5}+A_{34} \lambda^{4}+A_{33} \lambda^{3}+A_{32} \lambda^{2}+A_{31} \lambda+A_{30}\right) e^{\lambda \tau_{2}} \\
& +\left(C_{33} \lambda^{3}+C_{32} \lambda^{2}+C_{31} \lambda+C_{30}\right) e^{-\lambda \tau_{2}} \\
& +\left(D_{32} \lambda^{2}+D_{31} \lambda+D_{30}\right) e^{-2 \lambda \tau_{2}}=0
\end{aligned}
$$

Let $\lambda=i \omega_{2}\left(\omega_{2}>0\right)$ be the root of (24). Then,

$$
\begin{gathered}
g_{31}\left(\omega_{2}\right) \cos \tau_{2} \omega_{2}-g_{32}\left(\omega_{2}\right) \sin \tau_{2} \omega_{2}+g_{33}\left(\omega_{2}\right) \\
=h_{31}\left(\omega_{2}\right) \sin 2 \tau_{2} \omega_{2}+h_{32}\left(\omega_{2}\right) \cos 2 \tau_{2} \omega_{2}, \\
g_{34}\left(\omega_{2}\right) \sin \tau_{2} \omega_{2}+g_{35}\left(\omega_{2}\right) \cos \tau_{2} \omega_{2}+g_{36}\left(\omega_{2}\right) \\
=h_{31}\left(\omega_{2}\right) \cos 2 \tau_{2} \omega_{2}-h_{32}\left(\omega_{2}\right) \sin 2 \tau_{2} \omega_{2},
\end{gathered}
$$


where

$$
\begin{aligned}
& g_{31}\left(\omega_{2}\right)=A_{34} \omega_{2}^{4}-\left(A_{32}+C_{32}\right) \omega_{2}^{2}+A_{30}+C_{30}, \\
& g_{32}\left(\omega_{2}\right)=\omega_{2}^{5}-\left(A_{33}-C_{33}\right) \omega_{2}^{3}+\left(A_{31}-C_{31}\right) \omega_{2}, \\
& g_{33}\left(\omega_{2}\right)=B_{34} \omega_{2}^{4}-B_{32} \omega_{2}^{2}+B_{30}, \\
& g_{34}\left(\omega_{2}\right)=A_{34} \omega_{2}^{4}-\left(A_{32}-C_{32}\right) \omega_{2}^{2}+A_{30}-C_{30}, \\
& g_{35}\left(\omega_{2}\right)=\omega_{2}^{5}-\left(A_{33}+C_{33}\right) \omega_{2}^{3}+\left(A_{31}+C_{31}\right) \omega_{2}, \\
& g_{36}\left(\omega_{2}\right)=B_{31} \omega_{2}-B_{33} \omega_{2}^{3}, \\
& h_{31}\left(\omega_{2}\right)=-D_{31} \omega_{2}, \\
& h_{32}\left(\omega_{2}\right)=D_{32} \omega_{2}^{2}-D_{30} .
\end{aligned}
$$

According to the analysis in [11], we obtain the expressions of $\cos \tau_{2} \omega_{2}$ and $\sin \tau_{2} \omega_{2}$ when $\sin \tau_{2} \omega_{2}=$ $\sqrt{1-\cos ^{2} \tau_{2} \omega_{2}}$ and we denote $f_{31}\left(\omega_{2}\right)=\cos \tau_{2} \omega_{2}$ and $f_{32}\left(\omega_{2}\right)$ $=\sin \tau_{2} \omega_{2}$. Then, we obtain a function regarding $\omega_{2}$ :

$$
f_{31}^{2}\left(\omega_{2}\right)+f_{32}^{2}\left(\omega_{2}\right)=1 .
$$

Next, we suppose that

\section{$\left(H_{31}\right)$ Equation (27) has at least one positive root.}

Then, there exists $\omega_{210}>0$ which makes (24) have roots $\pm i \omega_{210}$. For $\omega_{210}$,

$$
\tau_{210}=\frac{1}{\omega_{210}} \arccos f_{31}\left(\omega_{210}\right) .
$$

Similarly, one can also obtain expressions of $\cos \tau_{2} \omega_{2}$ and $\sin \tau_{2} \omega_{2}$ for $\sin \tau_{2} \omega_{2}=-\sqrt{1-\cos ^{2} \tau_{2} \omega_{2}}$ and we denote $f_{33}\left(\omega_{2}\right)=\cos \tau_{2} \omega_{2}$ and $f_{34}\left(\omega_{2}\right)=\sin \tau_{2} \omega_{2}$. Then,

$$
f_{33}^{2}\left(\omega_{2}\right)+f_{34}^{2}\left(\omega_{2}\right)=1 .
$$

Obviously, if (29) has a root $\omega_{220}>0$, then (24) has roots $\pm i \omega_{220}$. For $\omega_{220}$,

$$
\tau_{220}=\frac{1}{\omega_{220}} \arccos f_{33}\left(\omega_{220}\right) .
$$

Let

$$
\tau_{20}=\min \left\{\tau_{210}, \tau_{220}\right\},
$$

and $\pm i \omega_{20}$ are the roots of (24) when $\tau_{2}=\tau_{20}$. Differentiating (24) regarding $\tau_{2}$, one can obtain

$$
\begin{aligned}
& {\left[\frac{d \lambda}{d \tau_{2}}\right]^{-1}} \\
& =\frac{p_{30}(\lambda)+p_{31}(\lambda) e^{\lambda \tau_{2}}+p_{32}(\lambda) e^{-\lambda \tau_{2}}+p_{33}(\lambda) e^{-2 \lambda \tau_{2}}}{q_{30}(\lambda)-q_{31}(\lambda) e^{\lambda \tau_{2}}} \\
& \quad-\frac{\tau_{2}}{\lambda},
\end{aligned}
$$

with

$$
\begin{aligned}
p_{30}(\lambda)= & 4 B_{34} \lambda^{3}+3 B_{33} \lambda^{2}+2 B_{32} \lambda+B_{31}, \\
p_{31}(\lambda)= & 5 \lambda^{4}+4 A_{34} \lambda^{3}+3 A_{33} \lambda^{2}+2 A_{32} \lambda+A_{31}, \\
p_{32}(\lambda)= & 3 C_{33} \lambda^{2}+2 C_{32} \lambda+C_{31}, \\
p_{33}(\lambda)= & 2 D_{32} \lambda+D_{31}, \\
q_{30}(\lambda)= & C_{33} \lambda^{4}+\left(C_{32}+2 D_{32}\right) \lambda^{3}+\left(C_{31}+2 D_{31}\right) \lambda^{2} \\
& +\left(C_{30}+2 D_{30}\right) \lambda, \\
& +A_{30} \lambda .
\end{aligned}
$$

Define

$$
\operatorname{Re}\left[\frac{d \lambda}{d \tau_{2}}\right]_{\lambda=i \omega_{20}}^{-1}=\frac{P_{3 R} Q_{3 R}+P_{3 I} Q_{3 I}}{Q_{3 R}^{2}+Q_{3 I}^{2}}
$$

Obviously, if the condition

$\left(H_{32}\right) P_{3 R} Q_{3 R}+P_{3 I} Q_{3 I} \neq 0$ holds, then $\operatorname{Re}\left[d \lambda / d \tau_{2}\right]_{\lambda=i \omega_{20}}^{-1} \neq 0$.

Therefore, we can otain the following according to the analysis above.

Theorem 2. For system (4), let $\tau_{20}$ be specified by (31). If the conditions $\left(H_{31}\right)-\left(H_{32}\right)$ are satisfied, then $D_{*}\left(S_{*}, E_{*}\right.$, $\left.I_{*}, R_{*}, V_{*}\right)$ is asymptotically stable when $\tau_{2} \in\left[0, \tau_{20}\right)$ and $a$ Hopf bifurcation occurs at $D_{*}\left(S_{*}, E_{*}, I_{*}, R_{*}, V_{*}\right)$ when $\tau_{2}=$ $\tau_{20}$.

Case $4\left(\tau_{1}>0, \tau_{2}>0\right.$, and $\left.\tau_{2} \in\left(0, \tau_{20}\right)\right)$. Let $\lambda=i \omega_{1 *}$ $\left(\omega_{1 *}>0\right)$ be the root of (6); then, one can obtain

$$
\begin{aligned}
& g_{41}\left(\omega_{1 *}\right) \sin \tau_{1} \omega_{1 *}+g_{42}\left(\omega_{1 *}\right) \cos \tau_{1} \omega_{1 *} \\
& =h_{41}\left(\omega_{1 *}\right), \\
& g_{41}\left(\omega_{1 *}\right) \cos \tau_{1} \omega_{1 *}-g_{42}\left(\omega_{1 *}\right) \sin \tau_{1} \omega_{1 *} \\
& =h_{42}\left(\omega_{1 *}\right),
\end{aligned}
$$

where

$$
\begin{aligned}
g_{41}\left(\omega_{1 *}\right)= & B_{1} \omega_{1 *}-B_{3} \omega_{1 *}^{3} \\
& +\left(E_{1} \omega_{1 *}-E_{3} \omega_{1 *}^{3}\right) \cos \tau_{2} \omega_{1 *} \\
& -\left(E_{0}-E_{2} \omega_{1 *}^{2}\right) \sin \tau_{2} \omega_{1 *} \\
& +F_{1} \omega_{1 *} \cos 2 \tau_{2} \omega_{1 *} \\
& -\left(F_{0}-F_{2} \omega_{1 *}^{2}\right) \sin 2 \tau_{2} \omega_{1 *} \\
& +H_{1} \omega_{1 *} \cos 3 \tau_{2} \omega_{1 *}-H_{0} \sin 3 \tau_{2} \omega_{1 *},
\end{aligned}
$$




$$
\begin{aligned}
g_{42}\left(\omega_{1 *}\right)= & B_{4} \omega_{1 *}^{4}-B_{2} \omega_{1 *}^{2}+B_{0} \\
& +\left(E_{1} \omega_{1 *}-E_{3} \omega_{1 *}^{3}\right) \sin \tau_{2} \omega_{1 *} \\
& +\left(E_{0}-E_{2} \omega_{1 *}^{2}\right) \cos \tau_{2} \omega_{1 *} \\
& +F_{1} \omega_{1 *} \sin 2 \tau_{2} \omega_{1 *} \\
& +\left(F_{0}-F_{2} \omega_{1 *}^{2}\right) \cos 2 \tau_{2} \omega_{1 *} \\
& +H_{1} \omega_{1 *} \sin 3 \tau_{2} \omega_{1 *}+H_{0} \cos 3 \tau_{2} \omega_{1 *} \\
h_{41}\left(\omega_{1 *}\right)= & A_{2} \omega_{1 *}^{2}-A_{4} \omega_{1 *}^{4}-A_{0} \\
& +\left(C_{3} \omega_{1 *}^{3}-C_{1} \omega_{1 *}\right) \sin \tau_{2} \omega_{1 *} \\
& +\left(C_{2} \omega_{1 *}^{2}-C_{4} \omega_{1 *}^{4}-C_{0}\right) \cos \tau_{2} \omega_{1 *} \\
& +\left(D_{3} \omega_{1 *}^{3}-D_{1} \omega_{1 *}\right) \sin 2 \tau_{2} \omega_{1 *} \\
& +\left(D_{2} \omega_{1 *}^{2}-D_{0}\right) \cos 2 \tau_{2} \omega_{1 *} \\
& -G_{1} \omega_{1 *} \sin 3 \tau_{2} \omega_{1 *} \\
& +\left(G_{2} \omega_{1 *}^{2}-G_{0}\right) \cos 3 \tau_{2} \omega_{1 *},
\end{aligned}
$$

$$
\begin{aligned}
h_{42}\left(\omega_{1 *}\right)= & A_{3} \omega_{1 *}^{3}-\omega_{1 *}^{5}-A_{1} \omega_{1 *} \\
& +\left(C_{3} \omega_{1 *}^{3}-C_{1} \omega_{1 *}\right) \cos \tau_{2} \omega_{1 *} \\
& -\left(C_{2} \omega_{1 *}^{2}-C_{4} \omega_{1 *}^{4}-C_{0}\right) \sin \tau_{2} \omega_{1 *} \\
& +\left(D_{3} \omega_{1 *}^{3}-D_{1} \omega_{1 *}\right) \cos 2 \tau_{2} \omega_{1 *} \\
& -\left(D_{2} \omega_{1 *}^{2}-D_{0}\right) \sin 2 \tau_{2} \omega_{1 *} \\
& -G_{1} \omega_{1 *} \cos 3 \tau_{2} \omega_{1 *} \\
& -\left(G_{2} \omega_{1 *}^{2}-G_{0}\right) \sin 3 \tau_{2} \omega_{1 *} .
\end{aligned}
$$

Thus, we can obtain

$$
\begin{aligned}
f_{40}\left(\omega_{1 *}\right)+2 f_{41}\left(\omega_{1 *}\right) \cos \tau_{2} \omega_{1 *} \\
\quad+2 f_{42}\left(\omega_{1 *}\right) \sin \tau_{2} \omega_{1 *}+2 f_{43}\left(\omega_{1 *}\right) \cos 2 \tau_{2} \omega_{1 *} \\
+2 f_{44}\left(\omega_{1 *}\right) \sin 2 \tau_{2} \omega_{1 *}+2 f_{45}\left(\omega_{1 *}\right) \cos 3 \tau_{2} \omega_{1 *} \\
+2 f_{46}\left(\omega_{1 *}\right) \sin 3 \tau_{2} \omega_{1 *}=0,
\end{aligned}
$$

where

$$
\begin{gathered}
f_{40}\left(\omega_{1 *}\right)=\omega_{1 *}^{10}+\left(A_{4}^{2}-B_{4}^{2}+C_{4}^{2}-2 A_{3}\right) \omega_{1 *}^{8}+\left(A_{3}^{2}\right. \\
-B_{3}^{2}+C_{3}^{2}+D_{3}^{2}-E_{3}^{2}-2 A_{2} A_{4}+2 B_{2} B_{4}-2 C_{2} C_{4} \\
\left.+2 A_{1}\right) \omega_{1 *}^{6}+\left(A_{2}^{2}-B_{2}^{2}+C_{2}^{2}+D_{2}^{2}-E_{2}^{2}-F_{2}^{2}+G_{2}^{2}\right. \\
+2 A_{0} A_{4}-2 A_{1} A_{3}-2 B_{0} B_{4}+2 B_{1} B_{3}+2 C_{0} C_{4}
\end{gathered}
$$

$$
\begin{aligned}
& \left.-2 C_{1} C_{3}-2 D_{1} D_{3}+2 E_{1} E_{3}\right) \omega_{1 *}^{4}+\left(A_{1}^{2}-B_{1}^{2}+C_{1}^{2}\right. \\
+ & D_{1}^{2}-E_{1}^{2}+G_{1}^{2}-H_{1}^{2}-2 A_{0} A_{2}-2 C_{0} C_{2}-2 D_{0} D_{2} \\
& \left.+2 B_{0} B_{2}+2 E_{0} E_{2}+2 F_{0} F_{2}-2 G_{0} G_{2}\right) \omega_{1 *}^{2}+A_{0}^{2} \\
& +C_{0}^{2}+D_{0}^{2}+G_{0}^{2}-B_{0}^{2}-E_{0}^{2}-F_{0}^{2}-H_{0}^{2} \\
f_{41} & \left(\omega_{1 *}\right)=\left(A_{4} C_{4}-C_{3}\right) \omega_{1 *}^{8}+\left(A_{3} C_{3}-A_{2} C_{4}\right. \\
& \left.-A_{4} C_{2}-B_{3} E_{3}+B_{4} E_{2}+C_{3} D_{3}-C_{4} D_{2}+C_{1}\right) \omega_{1 *}^{6} \\
& +\left(A_{0} C_{4}-A_{1} C_{3}+A_{2} C_{2}-A_{3} C_{1}+A_{4} C_{0}-C_{1} D_{3}\right. \\
& -C_{3} D_{1}+D_{2} G_{2}-D_{3} G_{1}+C_{2} D_{2}+C_{4} D_{0}+B_{1} E_{3} \\
& \left.-B_{2} E_{2}+B_{3} E_{1}-B_{4} E_{0}+E_{3} F_{1}-E_{2} F_{2}\right) \omega_{1 *}^{4} \\
& +\left(A_{1} C_{1}-A_{0} C_{2}-A_{2} C_{0}+C_{1} D_{1}-C_{0} D_{2}-C_{2} D_{0}\right. \\
& +D_{1} G_{1}-D_{0} G_{2}-D_{2} G_{0}-B_{1} E_{1}+B_{2} E_{0}+B_{0} E_{2} \\
& \left.-E_{1} F_{1}+E_{0} F_{2}+E_{2} F_{0}-F_{1} H_{1}+F_{2} H_{0}\right) \omega_{1 *}^{2}-B_{0} E_{0} \\
& -E_{0} F_{0}-F_{0} H_{0},
\end{aligned}
$$$$
f_{42}\left(\omega_{1 *}\right)=-C_{4} \omega_{1 *}^{9}+\left(A_{3} C_{4}-A_{4} C_{3}+B_{4} E_{3}\right.
$$$$
\left.-C_{4} D_{3}+C_{2}\right) \omega_{1 *}^{7}+\left(A_{2} C_{3}+A_{4} C_{1}-A_{3} C_{2}\right.
$$$$
-A_{1} C_{4}-B_{2} E_{3}+B_{3} E_{2}-B_{4} E_{1}+C_{2} D_{3}+C_{4} D_{1}
$$$$
\left.-C_{3} D_{2}-D_{3} G_{2}-E_{3} F_{2}-C_{0}\right) \omega_{1 *}^{5}+\left(A_{1} C_{2}\right.
$$$$
+A_{3} C_{0}-A_{2} C_{1}-A_{0} C_{3}+B_{0} E_{3}-B_{1} E_{2}+B_{2} E_{1}
$$$$
-B_{3} E_{0}-C_{0} D_{3}+C_{1} D_{2}-C_{2} D_{1}+D_{2} G_{1}+C_{3} D_{0}
$$$$
\left.-D_{1} G_{2}-D_{3} G_{0}-E_{1} F_{2}+E_{2} F_{1}+F_{2} H_{1}-E_{3} F_{0}\right)
$$$$
\cdot \omega_{1 *}^{3}+\left(A_{0} C_{1}-A_{1} C_{0}-B_{0} E_{1}-B_{1} E_{0}+C_{0} D_{1}\right.
$$$$
-D_{0} G_{1}-C_{1} D_{0}-D_{1} G_{0}-E_{0} F_{1}+E_{1} F_{0}-F_{0} H_{0}
$$$$
\left.+F_{1} H_{0}\right) \omega_{1 *} \text {, }
$$

$$
\begin{aligned}
& f_{43}\left(\omega_{1 *}\right)=-D_{3} \omega_{1 *}^{8}+\left(A_{3} D_{3}-A_{4} D_{2}+B_{4} F_{2}\right. \\
& \left.\quad-C_{4} G_{2}+D_{1}\right) \omega_{1 *}^{6}+\left(A_{2} D_{2}-A_{3} D_{1}+A_{4} D_{0}\right. \\
& \quad-A_{1} D_{3}-B_{2} F_{2}+B_{3} F_{1}-B_{4} F_{0}-C_{3} G_{1}+C_{4} G_{0} \\
& \left.\quad+C_{2} G_{2}-E_{3} H_{1}\right) \omega_{1 *}^{4}+\left(A_{1} D_{1}-A_{0} D_{2}-A_{2} D_{0}\right. \\
& \quad+B_{0} F_{2}+B_{2} F_{0}-B_{1} F_{1}-C_{0} G_{2}+C_{1} G_{1}-C_{2} G_{0} \\
& \left.\quad-E_{1} H_{1}+E_{2} H_{0}\right) \omega_{1 *}^{2}+A_{0} D_{0}+C_{0} G_{0}-B_{0} F_{0} \\
& \quad-E_{0} H_{0}, \\
& f_{44}\left(\omega_{1 *}\right)=\left(D_{2}-A_{4} D_{3}\right) \omega_{1 *}^{7}+\left(A_{2} D_{3}-A_{3} D_{2}\right. \\
& \left.+A_{4} D_{1}+B_{3} F_{2}-B_{4} F_{1}-C_{3} G_{2}+C_{4} G_{1}-D_{0}\right) \omega_{1 *}^{5} \\
& +\left(A_{1} D_{2}-A_{2} D_{1}+A_{3} D_{0}-A_{0} D_{3}-B_{1} F_{2}+B_{2} F_{1}\right.
\end{aligned}
$$




$$
\begin{gathered}
\left.-B_{3} F_{0}-C_{2} G_{1}+C_{1} G_{2}+C_{3} G_{0}+E_{2} H_{1}-E_{3} H_{0}\right) \\
\cdot \omega_{1 *}^{3}+\left(A_{0} D_{1}-A_{1} D_{0}+C_{0} G_{1}-C_{1} G_{0}+B_{1} F_{0}\right. \\
\left.-B_{0} F_{1}-E_{0} H_{1}+E_{1} H_{0}\right) \omega_{1 *}, \\
f_{45}\left(\omega_{1 *}\right)=\left(G_{1}-A_{4} G_{2}\right) \omega_{1 *}^{6}+\left(A_{2} G_{2}-A_{3} G_{1}\right. \\
\left.+A_{4} G_{0}+B_{3} H_{1}-B_{4} H_{0}\right) \omega_{1 *}^{4}+\left(A_{1} G_{1}-A_{0} G_{2}\right. \\
\left.-A_{2} G_{0}-B_{1} H_{1}+B_{2} H_{0}\right) \omega_{1 *}^{2}+A_{0} G_{0}-B_{0} H_{0}, \\
f_{46}\left(\omega_{1 *}\right)=G_{2} \omega_{1 *}^{7}+\left(A_{4} G_{1}-A_{3} G_{2}-B_{4} H_{1}-G_{0}\right) \\
\cdot \omega_{1 *}^{5}+\left(A_{1} G_{2}-A_{2} G_{1}+A_{3} G_{0}+B_{2} H_{1}-B_{3} H_{0}\right) \\
\cdot \omega_{1 *}^{3}+\left(A_{0} G_{1}-A_{1} G_{0}-B_{0} H_{1}+B_{1} H_{0}\right) \omega_{1 *} .
\end{gathered}
$$

\section{Suppose that}

\section{$\left(H_{41}\right)$ Equation (37) has at least one positive root.}

Then, there exists a positive root $\omega_{10}^{*}$ for (37) and (6) has roots $\pm i \omega_{10}^{*}$. For $\omega_{10}^{*}$, we have

$$
\begin{aligned}
& \tau_{10}^{*}=\frac{1}{\omega_{10}^{*}} \\
& \quad \times \arccos \frac{g_{41}\left(\omega_{10}^{*}\right) \times h_{42}\left(\omega_{10}^{*}\right)+g_{42}\left(\omega_{10}^{*}\right) \times h_{41}\left(\omega_{10}^{*}\right)}{g_{41}^{2}\left(\omega_{10}^{*}\right)+g_{42}^{2}\left(\omega_{10}^{*}\right)} .
\end{aligned}
$$

Differentiating (6) with respect to $\tau_{1}$, one can obtain

$$
\left[\frac{d \lambda}{d \tau_{1}}\right]^{-1}=\frac{P_{4 *}(\lambda)}{Q_{4 *}(\lambda)}-\frac{\tau_{1}}{\lambda}
$$

where

$$
\begin{aligned}
P_{4 *}(\lambda)= & p_{40}(\lambda)+p_{41}(\lambda) e^{-\lambda \tau_{1}}+p_{42}(\lambda) e^{-\lambda \tau_{2}} \\
& +p_{43}(\lambda) e^{-2 \lambda \tau_{2}}+p_{44}(\lambda) e^{-\lambda\left(\tau_{1}+\tau_{2}\right)} \\
& +p_{45}(\lambda) e^{-\lambda\left(\tau_{1}+2 \tau_{2}\right)}+p_{46}(\lambda) e^{-3 \lambda \tau_{2}} \\
& +p_{47}(\lambda) e^{-\lambda\left(\tau_{1}+3 \tau_{2}\right)} \\
Q_{4 *}(\lambda)= & q_{41}(\lambda) e^{-\lambda \tau_{1}}+q_{42}(\lambda) e^{-\lambda\left(\tau_{1}+\tau_{2}\right)} \\
& +q_{43}(\lambda) e^{-\lambda\left(\tau_{1}+2 \tau_{2}\right)}+q_{44}(\lambda) e^{-\lambda\left(\tau_{1}+3 \tau_{2}\right)},
\end{aligned}
$$

with

$$
\begin{aligned}
p_{40}(\lambda)= & 5 \lambda^{4}+4 A_{4} \lambda^{3}+3 A_{3} \lambda^{2}+2 A_{2} \lambda+A_{1}, \\
p_{41}(\lambda)= & 4 B_{4} \lambda^{3}+3 B_{3} \lambda^{2}+2 B_{2} \lambda+B_{1}, \\
p_{42}(\lambda)= & -\tau_{2} C_{4} \lambda^{4}+\left(4 C_{4}-\tau_{2} C_{3}\right) \lambda^{3} \\
& +\left(3 C_{3}-\tau_{2} C_{2}\right) \lambda^{2}+\left(2 C_{2}-\tau_{2} C_{1}\right) \lambda \\
& +C_{1}-\tau_{2} C_{0}, \\
p_{43}(\lambda)= & -2 \tau_{2} D_{3} \lambda^{3}+\left(3 D_{3}-2 \tau_{2} D_{2}\right) \lambda^{2} \\
& +\left(2 D_{2}-2 \tau_{2} D_{1}\right) \lambda+D_{1}-2 \tau_{2} D_{0}, \\
& +\left(2 E_{2}-\tau_{2} E_{1}\right) \lambda+E_{1}-\tau_{2} E_{0}, \\
p_{44}(\lambda)= & -\tau_{2} E_{3} \lambda^{3}+\left(3 E_{3}-\tau_{2} E_{2}\right) \lambda^{2} \\
p_{45}(\lambda)= & -2 \tau_{2} F_{2} \lambda^{2}+2\left(F_{2}-\tau_{2} F_{1}\right) \lambda+F_{1}-2 \tau_{2} F_{0}, \\
p_{46}(\lambda)= & -3 \tau_{2} G_{2} \lambda^{2}+\left(2 G_{2}-3 \tau_{2} G_{1}\right) \lambda+G_{1}-3 \tau_{2} G_{0}, \\
p_{47}(\lambda)= & -2 \tau_{2} H_{2} \lambda+H_{1}-3 \tau_{2} H_{0}, \\
q_{41}(\lambda)= & B_{4} \lambda^{5}+B_{3} \lambda^{4}+B_{2} \lambda^{3}+B_{1} \lambda^{2}+B_{0} \lambda, \\
q_{42}(\lambda)= & E_{3} \lambda^{4}+E_{2} \lambda^{3}+E_{1} \lambda^{2}+E_{0} \lambda \\
q_{43}(\lambda)= & F_{2} \lambda^{3}+F_{1} \lambda^{2}+F_{0} \lambda, \\
q_{44}(\lambda)= & H_{1} \lambda^{2}+H_{0} \lambda . \\
& \\
& \\
&
\end{aligned}
$$

Define

$$
\operatorname{Re}\left[\frac{d \lambda}{d \tau_{1}}\right]_{\lambda=i \omega_{10}^{*}}^{-1}=\frac{P_{4 R} Q_{4 R}+P_{4 I} Q_{4 I}}{Q_{4 R}^{2}+Q_{4 I}^{2}}
$$

Obviously, if the condition

$\left(H_{42}\right) P_{4 R} Q_{4 R}+P_{4 I} Q_{4 I} \neq 0$ holds, then $\operatorname{Re}\left[d \lambda / d \tau_{1}\right]_{\lambda=i \omega_{10}^{*}}^{-1} \neq 0$.

Thus, we have the following based on the analysis above.

Theorem 3. For system (4), let $\tau_{10}^{*}$ be specified by (39) and $\tau_{2} \in\left(0, \tau_{20}\right)$. If the conditions $\left(H_{41}\right)-\left(H_{42}\right)$ are satisfied, then $D_{*}\left(S_{*}, E_{*}, I_{*}, R_{*}, V_{*}\right)$ is asymptotically stable when $\tau_{1} \in$ $\left[0, \tau_{10}^{*}\right)$ and a Hopf bifurcation occurs at $D_{*}\left(S_{*}, E_{*}, I_{*}, R_{*}, V_{*}\right)$ when $\tau_{1}=\tau_{10}^{*}$.

Case $5\left(\tau_{1}>0, \tau_{2}>0\right.$, and $\left.\tau_{1} \in\left(0, \tau_{10}\right)\right)$. Multiplying by $e^{\lambda \tau_{2}}$, (6) becomes

$$
\begin{aligned}
& C_{4} \lambda^{4}+C_{3} \lambda^{3}+C_{2} \lambda^{2}+C_{1} \lambda+C_{0} \\
& \quad+\left(E_{3} \lambda^{3}+E_{2} \lambda^{2}+E_{1} \lambda+E_{0}\right) e^{-\lambda \tau_{1}} \\
& \quad+\left(\lambda^{5}+A_{4} \lambda^{4}+A_{3} \lambda^{3}+A_{2} \lambda^{2}+A_{1} \lambda+A_{0}\right) e^{\lambda \tau_{2}} \\
& \quad+\left(D_{3} \lambda^{3}+D_{2} \lambda^{2}+D_{1} \lambda+D_{0}\right) e^{-\lambda \tau_{2}}
\end{aligned}
$$




$$
\begin{aligned}
& +\left(B_{4} \lambda^{4}+B_{3} \lambda^{3}+B_{2} \lambda^{2}+B_{1} \lambda+B_{0}\right) e^{\lambda\left(\tau_{2}-\tau_{1}\right)} \\
& +\left(F_{2} \lambda^{2}+F_{1} \lambda+F_{0}\right) e^{-\lambda\left(\tau_{1}+\tau_{2}\right)}
\end{aligned}
$$

Let $\lambda=i \omega_{2 *}\left(\omega_{2 *}>0\right)$ be a root of (44). Then,

$$
\begin{gathered}
g_{51}\left(\omega_{2 *}\right) \cos \tau_{2} \omega_{2 *}-g_{52}\left(\omega_{2 *}\right) \sin \tau_{2} \omega_{2 *}+g_{53}\left(\omega_{2 *}\right) \\
=h_{51}\left(\omega_{2 *}\right) \sin 2 \tau_{2} \omega_{2 *}+h_{52}\left(\omega_{2 *}\right) \cos 2 \tau_{2} \omega_{2 *}, \\
g_{54}\left(\omega_{2 *}\right) \sin \tau_{2} \omega_{2 *}+g_{55}\left(\omega_{2 *}\right) \cos \tau_{2} \omega_{2 *}+g_{56}\left(\omega_{2 *}\right) \\
=h_{51}\left(\omega_{2 *}\right) \cos 2 \tau_{2} \omega_{2 *}-h_{52}\left(\omega_{2 *}\right) \sin 2 \tau_{2} \omega_{2 *},
\end{gathered}
$$

where

$$
\begin{aligned}
g_{51}\left(\omega_{2 *}\right) & \\
= & A_{4} \omega_{2 *}^{4}-\left(A_{2}+D_{2}\right) \omega_{2 *}^{2}+A_{0}+D_{0} \\
& +\left(B_{4} \omega_{2 *}^{4}-\left(B_{2}+F_{2}\right) \omega_{2 *}^{2}+B_{0}+F_{0}\right) \cos \tau_{1} \omega_{2 *} \\
& +\left(\left(B_{1}+F_{1}\right) \omega_{2 *}-B_{3} \omega_{2 *}^{3}\right) \sin \tau_{1} \omega_{2 *}, \\
g_{52}\left(\omega_{2 *}\right) & \\
= & \omega_{2 *}^{5}-\left(A_{3}-D_{3}\right) \omega_{2 *}^{3}+\left(A_{1}-D_{1}\right) \omega_{2 *} \\
& +\left(\left(B_{2}-F_{2}\right) \omega_{2 *}^{2}-B_{4} \omega_{2 *}^{4}-B_{0}+F_{0}\right) \sin \tau_{1} \omega_{2 *} \\
& +\left(\left(B_{1}-F_{1}\right) \omega_{2 *}-B_{3} \omega_{2 *}^{3}\right) \cos \tau_{1} \omega_{2 *}, \\
g_{53}\left(\omega_{2 *}\right) & \\
= & C_{4} \omega_{2 *}^{4}-C_{2} \omega_{2 *}^{2}+C_{0} \\
& +\left(E_{1} \omega_{2 *}-E_{3} \omega_{2 *}^{3}\right) \sin \tau_{1} \omega_{2 *} \\
& +\left(E_{0}-E_{2} \omega_{2 *}^{2}\right) \cos \tau_{1} \omega_{2 *},
\end{aligned}
$$$$
g_{54}\left(\omega_{2 *}\right)
$$$$
=A_{4} \omega_{2 *}^{4}-\left(A_{2}-D_{2}\right) \omega_{2 *}^{2}+A_{0}-D_{0}
$$$$
+\left(B_{4} \omega_{2 *}^{4}-\left(B_{2}-F_{2}\right) \omega_{2 *}^{2}+B_{0}-F_{0}\right) \cos \tau_{1} \omega_{2 *}
$$$$
+\left(\left(B_{1}-F_{1}\right) \omega_{2 *}-B_{3} \omega_{2 *}^{3}\right) \sin \tau_{1} \omega_{2 *},
$$$$
g_{55}\left(\omega_{2 *}\right)
$$$$
=\omega_{2 *}^{5}-\left(A_{3}+D_{3}\right) \omega_{2 *}^{3}+\left(A_{1}+D_{1}\right) \omega_{2 *}
$$$$
+\left(\left(B_{2}+F_{2}\right) \omega_{2 *}^{2}-B_{4} \omega_{2 *}^{2}-B_{0}-F_{0}\right) \sin \tau_{1} \omega_{2 *}
$$$$
+\left(\left(B_{1}+F_{1}\right) \omega_{2 *}-B_{3} \omega_{2 *}^{3}\right) \cos \tau_{1} \omega_{2 *},
$$$$
g_{56}\left(\omega_{2 *}\right)
$$

$$
\begin{aligned}
= & C_{1} \omega_{2 *}-C_{3} \omega_{2 *}^{3}+\left(E_{1} \omega_{2 *}-E_{3} \omega_{2 *}^{3}\right) \cos \tau_{1} \omega_{2 *} \\
& -\left(E_{0}-E_{2} \omega_{2 *}^{2}\right) \sin \tau_{1} \omega_{2 *},
\end{aligned}
$$

$$
\begin{aligned}
& h_{51}\left(\omega_{2 *}\right) \\
& \quad=G_{1} \omega_{2 *}+H_{0} \sin \tau_{1} \omega_{2 *}-H_{1} \omega_{2 *} \cos \tau_{1} \omega_{2 *}, \\
& h_{52}\left(\omega_{2 *}\right) \\
& \quad=G_{0}-G_{2} \omega_{2 *}^{2}-H_{0} \cos \tau_{1} \omega_{2 *}-H_{1} \omega_{2 *} \sin \tau_{1} \omega_{2 *} .
\end{aligned}
$$

Similar to Case 3, we can obtain the expressions of $\cos \tau_{2} \omega_{2 *}$ and $\sin \tau_{2} \omega_{2 *}$ when $\sin \tau_{2} \omega_{2 *}=\sqrt{1-\cos ^{2} \tau_{2} \omega_{2 *}}$ and we denote $f_{51}\left(\omega_{2 *}\right)=\cos \tau_{2} \omega_{2 *}$ and $f_{52}\left(\omega_{2 *}\right)=$ $\sin \tau_{2} \omega_{2 *}$. Then, one can obtain

$$
f_{51}^{2}\left(\omega_{2 *}\right)+f_{52}^{2}\left(\omega_{2 *}\right)=1 .
$$

In order to give the main results in this paper, we suppose that $\left(H_{51}\right)$ Equation (47) has at least one positive root.

If the condition $\left(H_{51}\right)$ holds, then there exists $\omega_{21 *}>0$ such that (6) has a pair of purely imaginary roots $\pm i \omega_{21 *}$. For $\omega_{21 *}$, we have

$$
\tau_{21 *}=\frac{1}{\omega_{21 *}} \times \arccos f_{51}\left(\omega_{21 *}\right) .
$$

Similarly, one can obtain expressions of $\cos \tau_{2} \omega_{2 *}$ and $\sin \tau_{2} \omega_{2 *}$ when $\sin \tau_{2} \omega_{2 *}=-\sqrt{1-\cos ^{2} \tau_{2} \omega_{2 *}}$ and we denote $f_{53}\left(\omega_{2 *}\right)=\cos \tau_{2} \omega_{2 *}$ and $f_{54}\left(\omega_{2 *}\right)=\sin \tau_{2} \omega_{2 *}$. Then, one can obtain

$$
f_{53}^{2}\left(\omega_{2 *}\right)+f_{54}^{2}\left(\omega_{2 *}\right)=1 .
$$

If (49) has one positive root $\omega_{22 *}$, then (6) has roots $\pm i \omega_{22 *}$. For $\omega_{22 *}$, we have

$$
\tau_{22 *}=\frac{1}{\omega_{22 *}} \times \arccos f_{53}\left(\omega_{22 *}\right) .
$$

Let

$$
\tau_{20}^{*}=\min \left\{\tau_{21 *}, \tau_{22 *}\right\}
$$

and $\pm i \omega_{20}^{*}$ be the roots of (6) with $\tau_{2}=\tau_{20}^{*}$. Differentiating (6) with respect to $\tau_{2}$, we get

$$
\left[\frac{d \lambda}{d \tau_{2}}\right]^{-1}=\frac{P_{5 *}(\lambda)}{Q_{5 *}(\lambda)}-\frac{\tau_{2}}{\lambda}
$$

where

$$
\begin{aligned}
P_{5 *}(\lambda)= & p_{50}(\lambda)+p_{51}(\lambda) e^{-\lambda \tau_{1}}+p_{52}(\lambda) e^{-\lambda \tau_{2}} \\
& +p_{53}(\lambda) e^{-2 \lambda \tau_{2}}+p_{54}(\lambda) e^{-\lambda\left(\tau_{1}+\tau_{2}\right)} \\
& +p_{55}(\lambda) e^{-\left(\lambda \tau_{1}+2 \tau_{2}\right)}+p_{56}(\lambda) e^{-3 \lambda \tau_{2}} \\
& +p_{57}(\lambda) e^{-\lambda\left(\tau_{1}+3 \tau_{2}\right)} \\
Q_{5 *}(\lambda)= & q_{51}(\lambda) e^{-\lambda \tau_{2}}+q_{52}(\lambda) e^{-\lambda\left(\tau_{1}+\tau_{2}\right)} \\
& +q_{53}(\lambda) e^{-2 \lambda \tau_{2}}+q_{54}(\lambda) e^{-\lambda\left(\tau_{1}+\tau_{2}\right)} \\
& +q_{55}(\lambda) e^{-3 \lambda \tau_{2}}+q_{56}(\lambda) e^{-\lambda\left(\tau_{1}+3 \tau_{2}\right)},
\end{aligned}
$$


with

$$
\begin{aligned}
& p_{50}(\lambda)= 5 \lambda^{4}+4 A_{4} \lambda^{3}+3 A_{3} \lambda^{2}+2 A_{2} \lambda+A_{1}, \\
& p_{51}(\lambda)=-\tau_{1} B_{4} \lambda^{4}+\left(4 B_{4}-\tau_{1}-B_{3}\right) \lambda^{3} \\
&+\left(3 B_{3}-\tau_{1} B_{2}\right) \lambda^{2}+\left(2 B_{2}-\tau_{1} B_{1}\right)+B_{1} \\
&-\tau_{1} B_{0}, \\
& p_{52}(\lambda)= 4 C_{4} \lambda^{3}+3 C_{3} \lambda^{2}+2 C_{2} \lambda+C_{1}, \\
& p_{53}(\lambda)= 3 D_{3} \lambda^{2}+2 D_{2} \lambda+D_{1}, \\
& p_{54}(\lambda)=-\tau_{1} E_{3} \lambda^{3}+\left(3 E_{3}-\tau_{1} E_{2}\right) \lambda^{2} \\
&+\left(2 E_{2}-\tau_{1} E_{1}\right) \lambda+E_{1}-\tau_{1} E_{0}, \\
& p_{55}(\lambda)=-\tau_{1} F_{2} \lambda^{2}+\left(2 F_{2}-\tau_{1} F_{1}\right) \lambda+F_{1}-\tau_{1} F_{0}, \\
& p_{56}(\lambda)= 2 G_{2} \lambda+G_{1}, \\
& p_{57}(\lambda)=-\tau_{1} H_{1} \lambda+H_{1}-\tau_{1}-H_{0}, \\
& q_{51}(\lambda)= C_{4} \lambda^{5}+C_{3} \lambda^{4}+C_{2} \lambda^{3}+C_{1} \lambda^{2}+C_{0} \lambda, \\
& q_{52}(\lambda)= E_{3} \lambda^{4}+E_{2} \lambda^{3}+E_{1} \lambda^{2}+E_{0} \lambda, \\
& q_{53}(\lambda)=2\left(D_{3} \lambda^{4}+D_{2} \lambda^{3}+D_{1} \lambda^{2}+D_{0} \lambda\right), \\
& q_{54}(\lambda)=2\left(F_{2} \lambda^{3}+F_{1} \lambda^{2}+F_{0} \lambda\right), \\
& q_{55}(\lambda)=3\left(G_{2} \lambda^{3}+G_{1} \lambda^{2}+G_{0} \lambda\right), \\
& q_{56}(\lambda)=3\left(H_{1} \lambda^{2}+H_{0} \lambda\right) . \\
&\left.p_{5}\right)
\end{aligned}
$$

Define

$$
\operatorname{Re}\left[\frac{d \lambda}{d \tau_{2}}\right]_{\lambda=i \omega_{20}^{*}}^{-1}=\frac{P_{5 R} Q_{5 R}+P_{5 I} Q_{5 I}}{Q_{5 R}^{2}+Q_{5 I}^{2}} .
$$

Obviously, if the condition

$\left(H_{52}\right) P_{5 R} Q_{5 R}+P_{5 I} Q_{5 I} \neq 0$ holds, then $\operatorname{Re}\left[d \lambda / d \tau_{2}\right]_{\lambda=i \omega_{20}^{*}}^{-1} \neq 0$.

Thus, we have the following based on the analysis above.

Theorem 4. For system (4), let $\tau_{20}^{*}$ be specified by (51) and $\tau_{1} \in\left(0, \tau_{10}\right)$. If the conditions $\left(H_{51}\right)-\left(H_{52}\right)$ are satisfied, then $D_{*}\left(S_{*}, E_{*}, I_{*}, R_{*}, V_{*}\right)$ is asymptotically stable when $\tau_{2} \in$ $\left[0, \tau_{20}^{*}\right)$ and a Hopf bifurcation occurs at $D_{*}\left(S_{*}, E_{*}, I_{*}, R_{*}, V_{*}\right)$ when $\tau_{2}=\tau_{20}^{*}$.

\section{Properties of the Hopf Bifurcation}

We will investigate properties of the Hopf bifurcation of system (4) with respect to $\tau_{1}$ and $\tau_{2} \in\left(0, \tau_{20}\right)$ in this section. Let $\tau_{2 *}<\tau_{10}^{*}$ with $\tau_{2 *} \in\left(0, \tau_{20}\right)$ and $\tau_{1}=\tau_{10}^{*}+\mu, \mu \in R$. By the transformation $u_{1}(t)=S(t)-S_{*}, u_{2}(t)=E(t)-E_{*}$, $u_{3}(t)=I(t)-I_{*}, u_{4}(t)=R(t)-R_{*}, u_{5}(t)=V(t)-V_{*}$, and $t \rightarrow\left(t / \tau_{1}\right)$, system (4) can be written as

$$
\dot{u}(t)=L_{\mu} u_{t}+F\left(\mu, u_{t}\right),
$$

where $u_{t}=\left(u_{1}(t), u_{2}(t), u_{3}(t), u_{4}(t), u_{5}(t)\right)^{T} \in C\left([-1,0], R^{5}\right)$, and

$$
\begin{aligned}
& L_{\mu} \phi=\left(\tau_{0}+\mu\right) \\
& \cdot\left(A_{\max } \phi(0)+B_{\max 1} \phi\left(-\frac{\tau_{2 *}}{\tau_{10}^{*}}\right)+B_{\max 2} \phi(-1)\right), \\
& F(\mu, \phi)=\left(\tau_{10}^{*}+\mu\right)\left(\begin{array}{c}
-\beta \phi_{1}(0) \phi_{3}(0) \\
\beta \phi_{1}(0) \phi_{3}(0) \\
0 \\
0 \\
0
\end{array}\right)
\end{aligned}
$$

where

$$
\begin{aligned}
A_{\max } & =\left(\begin{array}{ccccc}
a_{11} & 0 & a_{13} & 0 & 0 \\
a_{21} & a_{22} & a_{23} & 0 & 0 \\
0 & a_{32} & a_{33} & 0 & 0 \\
0 & 0 & 0 & a_{44} & 0 \\
a_{51} & 0 & 0 & 0 & a_{55}
\end{array}\right), \\
B_{\max 1} & =\left(\begin{array}{ccccc}
0 & 0 & 0 & b_{14} & b_{15} \\
0 & 0 & 0 & 0 & 0 \\
0 & 0 & b_{33} & 0 & 0 \\
0 & 0 & b_{43} & b_{44} & 0 \\
0 & 0 & 0 & 0 & b_{55}
\end{array}\right),
\end{aligned}
$$

$$
B_{\max 2}=\left(\begin{array}{ccccc}
0 & 0 & 0 & 0 & 0 \\
0 & b_{22} & 0 & 0 & 0 \\
0 & b_{32} & 0 & 0 & 0 \\
0 & 0 & 0 & 0 & 0 \\
0 & 0 & 0 & 0 & 0
\end{array}\right) .
$$

According to the Riesz representation theorem, we know that there exists a $5 \times 5$ matrix function $\eta(\theta, \mu):[-1,0] \rightarrow$ $R^{5 \times 5}$ such that

$$
L_{\mu} \phi=\int_{-1}^{0} d \eta(\theta, \mu) \phi(\theta), \quad \phi \in C .
$$

In fact, we choose

$$
\begin{aligned}
& \eta(\theta, \mu) \\
& = \begin{cases}\left(\tau_{10}^{*}+\mu\right)\left(A_{\max }+B_{\max 1}+B_{\max 2}\right), & \theta=0, \\
\left(\tau_{10}^{*}+\mu\right)\left(B_{\max 1}+B_{\max 2}\right), & \theta \in\left[-\frac{\tau_{2 *}}{\tau_{10}^{*}}, 0\right), \\
\left(\tau_{10}^{*}+\mu\right) B_{\max 2}, & \theta \in\left(-1,-\frac{\tau_{2 *}}{\tau_{10}^{*}}\right), \\
0, & \theta=-1 .\end{cases}
\end{aligned}
$$


For $\phi \in C\left([-1,0], R^{5}\right)$, we define

$$
\begin{aligned}
& A(\mu) \phi= \begin{cases}\frac{d \phi(\theta)}{d \theta}, & -1 \leq \theta<0, \\
\int_{-1}^{0} d \eta(\theta, \mu) \phi(\theta), & \theta=0,\end{cases} \\
& R(\mu) \phi= \begin{cases}0, & -1 \leq \theta<0, \\
F(\mu, \phi), & \theta=0 .\end{cases}
\end{aligned}
$$

Then, system (56) is equivalent to

$$
\dot{u}(t)=A(\mu) u_{t}+R(\mu) u_{t} \text {, }
$$

where $u_{t}(\theta)=u(t+\theta)$ for $\theta \in[-1,0]$.
Then, we define $A^{*}$ :

$$
A^{*}(\varphi)= \begin{cases}-\frac{d \varphi(s)}{d s}, & 0<s \leq 1, \\ \int_{-1}^{0} d \eta^{T}(s, 0) \varphi(-s), & s=0,\end{cases}
$$

and a bilinear form

$$
\begin{aligned}
\langle\varphi(s), \phi(\theta)\rangle= & \bar{\varphi}(0) \phi(0) \\
& -\int_{\theta=-1}^{0} \int_{\xi=0}^{\theta} \bar{\varphi}(\xi-\theta) d \eta(\theta) \phi(\xi) d \xi,
\end{aligned}
$$

where $\eta(\theta)=\eta(\theta, 0)$.

Let $\rho(\theta)=\left(1, \rho_{2}, \rho_{3}, \rho_{4}, \rho_{5}\right)^{T} e^{i \omega_{10}^{*} \tau_{10}^{*} \theta}$ be the eigenvector of $A(0)$ with $+i \omega_{10}^{*} \tau_{10}^{*}$ and let $\rho^{*}(s)=(1 / K)\left(1, \rho_{2}^{*}, \rho_{3}^{*}\right.$, $\left.\rho_{4}^{*}, \rho_{5}^{*}\right) e^{i \omega_{10}^{*} \tau_{10}^{*} s}$ be the eigenvector of $A^{*}(0)$ with $-i \omega_{10}^{*} \tau_{10}^{*}$. Then, we obtain

$$
\begin{aligned}
& \rho_{2}=\frac{a_{21}\left(i \omega_{10}^{*}-a_{33}-b_{33} e^{-i \tau_{2 *} \omega_{10}^{*}}\right)}{\left(i \omega_{10}^{*}-a_{22}-b_{22} e^{-i \tau_{10}^{*} \omega_{10}^{*}}\right)\left(i \omega_{10}^{*}-a_{33}-b_{33} e^{-\tau_{2 *} \omega_{10}^{*}}\right)-a_{23} b_{32} e^{-i \tau_{10}^{*} \omega_{10}^{*}}}, \\
& \rho_{3}=\frac{b_{32} e^{-i \tau_{10}^{*} \omega_{10}^{*}}}{i \omega_{10}^{*}-a_{33}-b_{33} e^{-\tau_{2 *} \omega_{10}^{*}}} \rho_{2} \\
& \rho_{4}=\frac{b_{43} e^{-i \tau_{2 *} \omega_{10}^{*}}}{i \omega_{10}^{*}-a_{44}-b_{44} e^{-i \tau_{2 *} \omega_{10}^{*}}} \rho_{3} \text {, } \\
& \rho_{5}=\frac{a_{51}}{\omega_{10}^{*}-a_{55}-b_{55} e^{-\tau_{2 *} \omega_{10}^{*}}}, \\
& \rho_{2}^{*}=-\frac{i \omega_{10}^{*}+a_{11}}{a_{21}}+\frac{a_{51} b_{15} e^{i \tau_{2 *} \omega_{10}^{*}}}{a_{21}\left(\omega_{10}^{*}+a_{55}+b_{55} e^{i \tau_{2 *} \omega_{10}^{*}}\right)} \text {, } \\
& \rho_{3}^{*}=\frac{\left(i \omega_{10}^{*}+a_{11}\right)\left(i \omega_{10}^{*}+a_{22}+b_{22} e^{-i \tau_{10}^{*}} \omega_{10}^{*}\right)}{a_{21} b_{32} e^{-i \tau_{10}^{*} \omega_{10}^{*}}}-\frac{a_{51} b_{15} e^{i \tau_{2 *} \omega_{10}^{*}}\left(i \omega_{10}^{*}+a_{22}+b_{22} e^{-i \tau_{10}^{*} \omega_{10}^{*}}\right)}{a_{21} b_{32} e^{-i \tau_{10}^{*} \omega_{10}^{*}}\left(i \omega_{10}^{*}+a_{55}+b_{55} e^{i \tau_{2 *} \omega_{10}^{*}}\right)}, \\
& \rho_{4}^{*}=-\frac{b_{14} e^{i \tau_{2 *} \omega_{10}^{*}}}{i \omega_{10}^{*}+a_{44}+b_{44} e^{i \tau_{2 *} \omega_{10}^{*}}}, \\
& \rho_{5}^{*}=-\frac{b_{15} e^{-i \tau_{2 *} \omega_{10}^{*}}}{i \omega_{140}^{*}+a_{55}+b_{55} e^{-i \tau_{2 *} \omega_{10}^{*}}} .
\end{aligned}
$$

From (64), we obtain

$$
\begin{aligned}
& \left\langle\rho^{*}, \rho\right\rangle=\frac{1}{\bar{K}}\left[1+\rho_{2} \bar{\rho}_{2}^{*}+\rho_{3} \bar{\rho}_{3}^{*}+\rho_{4} \bar{\rho}_{4}^{*}+\rho_{5} \bar{\rho}_{5}^{*}\right. \\
& +\tau_{10}^{*} e^{-i \tau_{10}^{*} \omega_{10}^{*}} \rho_{2}\left(b_{22} \overline{\rho_{2}^{*}}+b_{32} \overline{\rho_{3}^{*}}\right) \\
& +\tau_{2 *} e^{-i \tau_{2 *} \omega_{10}^{*}}\left(\rho_{3}\left(b_{33} \bar{\rho}_{3}^{*}+b_{43} \bar{\rho}_{4}^{*}\right)\right. \\
& \left.\left.+\rho_{4}\left(b_{14}+b_{44} \bar{\rho}_{4}^{*}\right)+\rho_{5}\left(b_{15}+b_{55} \bar{\rho}_{5}^{*}\right)\right)\right] .
\end{aligned}
$$

Then, we can choose

$$
\begin{aligned}
\bar{K} & =1+\rho_{2} \bar{\rho}_{2}^{*}+\rho_{3} \bar{\rho}_{3}^{*}+\rho_{4} \bar{\rho}_{4}^{*}+\rho_{5} \bar{\rho}_{5}^{*} \\
& +\tau_{10}^{*} e^{-i \tau_{10}^{*} \omega_{10}^{*}} \rho_{2}\left(b_{22} \overline{\rho_{2}^{*}}+b_{32} \overline{\rho_{3}^{*}}\right) \\
& +\tau_{2 *} e^{-i \tau_{2 *} \omega_{10}^{*}}\left(\rho_{3}\left(b_{33} \bar{\rho}_{3}^{*}+b_{43} \bar{\rho}_{4}^{*}\right)\right. \\
& \left.+\rho_{4}\left(b_{14}+b_{44} \bar{\rho}_{4}^{*}\right)+\rho_{5}\left(b_{15}+b_{55} \bar{\rho}_{5}^{*}\right)\right) .
\end{aligned}
$$

such that $\left\langle\rho^{*}, \rho\right\rangle=1$ and $\left\langle\rho^{*}, \bar{\rho}\right\rangle=0$. 
Following the same algorithms introduced in [17] and the similar computation used in [18-22], we obtain

$$
\begin{aligned}
& g_{20}=2 \beta \tau_{10}^{*} \bar{K} \rho_{3}\left(\bar{\rho}_{2}^{*}-1\right), \\
& g_{11}=\beta \tau_{10}^{*} \bar{K}\left(\rho_{3}+\bar{\rho}_{3}\right)\left(\bar{\rho}_{2}^{*}-1\right), \\
& g_{02}=2 \beta \tau_{10}^{*} \bar{K} \bar{\rho}_{3}\left(\bar{\rho}_{2}^{*}-1\right),
\end{aligned}
$$

$$
\begin{aligned}
g_{21} & =2 \beta \tau_{10}^{*} \bar{K}\left(\bar{\rho}_{2}^{*}-1\right)\left(W_{11}^{(1)}(0) \rho_{3}+\frac{1}{2} W_{20}^{(1)}(0) \bar{\rho}_{3}\right. \\
& \left.+W_{11}^{(3)}(0)+\frac{1}{2} W_{20}^{(3)}(0)\right)
\end{aligned}
$$

with

$$
\begin{aligned}
& W_{20}(\theta)=\frac{i g_{20} \rho(0)}{\tau_{10}^{*} \omega_{10}^{*}} e^{i \tau_{10}^{*} \omega_{10}^{*} \theta}+\frac{i \bar{g}_{02} \bar{\rho}(0)}{3 \tau_{10}^{*} \omega_{10}^{*}} e^{-i \tau_{10}^{*} \omega_{10}^{*} \theta}+E_{1} e^{2 i \tau_{10}^{*} \omega_{10}^{*} \theta}, \\
& W_{11}(\theta)=-\frac{i g_{11} \rho(0)}{\tau_{10}^{*} \omega_{10}^{*}} e^{i \tau_{10}^{*} \omega_{10}^{*} \theta}+\frac{i \bar{g}_{11} \bar{\rho}(0)}{\tau_{10}^{*} \omega_{10}^{*}} e^{-i \tau_{10}^{*} \omega_{10}^{*} \theta}+E_{2} \text {, } \\
& E_{1}=2\left(\begin{array}{ccccc}
a_{11}^{\prime} & 0 & -a_{13} & -b_{14} e^{-2 i \tau_{2 *} \omega_{10}^{*}} & -b_{15} e^{-2 i \tau_{2 *} \omega_{10}^{*}} \\
-a_{21} & a_{22}^{\prime} & -a_{23} & 0 & 0 \\
0 & -a_{32}-b_{32} e^{-2 i \tau_{10}^{*} \omega_{10}^{*}} & a_{33}^{\prime} & 0 & 0 \\
0 & 0 & -b_{43} e^{-2 i \tau_{2 *} \omega_{10}^{*}} & a_{44}^{\prime} & 0 \\
-a_{51} & 0 & 0 & 0 & a_{55}^{\prime}
\end{array}\right)\left(\begin{array}{c}
-\beta \rho_{3} \\
\beta \rho_{3} \\
0 \\
0 \\
0
\end{array}\right) \text {, } \\
& E_{2}=-\left(\begin{array}{ccccc}
a_{11} & 0 & a_{13} & b_{14} & b_{15} \\
a_{21} & a_{22}+b_{22} & a_{23} & 0 & 0 \\
0 & a_{32}+b_{32} & a_{33}+b_{33} & 0 & 0 \\
0 & 0 & b_{43} & a_{44}+b_{44} & 0 \\
a_{51} & 0 & 0 & 0 & a_{55}+b_{55}
\end{array}\right) \quad\left(\begin{array}{c}
-\beta\left(\rho_{3}+\bar{\rho}_{3}\right) \\
\beta\left(\rho_{3}+\bar{\rho}_{3}\right) \\
0 \\
0 \\
0
\end{array}\right),
\end{aligned}
$$

where

$$
\begin{aligned}
& a_{11}^{\prime}=2 i \omega_{10}^{*}-a_{11}, \\
& a_{22}^{\prime}=2 i \omega_{10}^{*}-a_{22}-b_{22} e^{-2 i \omega_{10}^{*} \tau_{10}^{*},} \\
& a_{33}^{\prime}=2 i \omega_{10}^{*}-a_{33}-b_{33} e^{-2 i \omega_{10}^{*} \tau_{2 *},} \\
& a_{44}^{\prime}=2 i \omega_{10}^{*}-a_{44}-b_{44} e^{-2 i \omega_{10}^{*} \tau_{2 *}}, \\
& a_{55}^{\prime}=2 i \omega_{10}^{*}-a_{55}-b_{55} e^{-2 i \omega_{10}^{*} \tau_{2 *}} .
\end{aligned}
$$

Consequently, we can compute the following values:

$$
\begin{aligned}
C_{1}(0) & =\frac{i}{2 \tau_{10}^{*} \omega_{10}^{*}}\left(g_{11} g_{20}-2\left|g_{11}\right|^{2}-\frac{\left|g_{02}\right|^{2}}{3}\right)+\frac{g_{21}}{2} \\
\mu_{2} & =-\frac{\operatorname{Re}\left\{C_{1}(0)\right\}}{\operatorname{Re}\left\{\lambda^{\prime}\left(\tau_{10}^{*}\right)\right\}} \\
\beta_{2} & =2 \operatorname{Re}\left\{C_{1}(0)\right\} \\
T_{2} & =-\frac{\operatorname{Im}\left\{C_{1}(0)\right\}+\mu_{2} \operatorname{Im}\left\{\lambda^{\prime}\left(\tau_{10}^{*}\right)\right\}}{\tau_{10}^{*} \omega_{10}^{*}} .
\end{aligned}
$$

According to the analysis about properties of the Hopf bifurcation in [17], we have the following for system (4).

Theorem 5. Let $\mu_{2}, \beta_{2}$, and $T_{2}$ be specified by (71). $\mu_{2}$ determines the direction of the Hopf bifurcation (supercritical if $\mu_{2}>0$, subcritical if $\mu_{2}<0$ ); $\beta_{2}$ determines the stability of the bifurcating periodic solutions (stable if $\beta<0$, unstable if $\beta>0) ; T_{2}>0$ determines the period of the bifurcating periodic solutions (increasing if $T_{2}>0$, decreasing if $T_{2}<0$ ).

\section{A Numerical Example}

In this section we give a numerical example of system (4) to validate the analysis above. By extracting the same values from [11], we get the following system:

$$
\begin{aligned}
\frac{d S(t)}{d t}= & 2-0.3 S(t) I(t)-0.323 S(t)+0.3 R\left(t-\tau_{2}\right) \\
& +0.06 V\left(t-\tau_{2}\right), \\
\frac{d E(t)}{d t}= & 0.3 S(t) I(t)-0.003 E(t)-0.25 E\left(t-\tau_{1}\right), \\
\frac{d I(t)}{d \mathrm{t}}= & 0.25 E\left(t-\tau_{1}\right)-0.073 I(t)-0.4 I\left(t-\tau_{2}\right),
\end{aligned}
$$




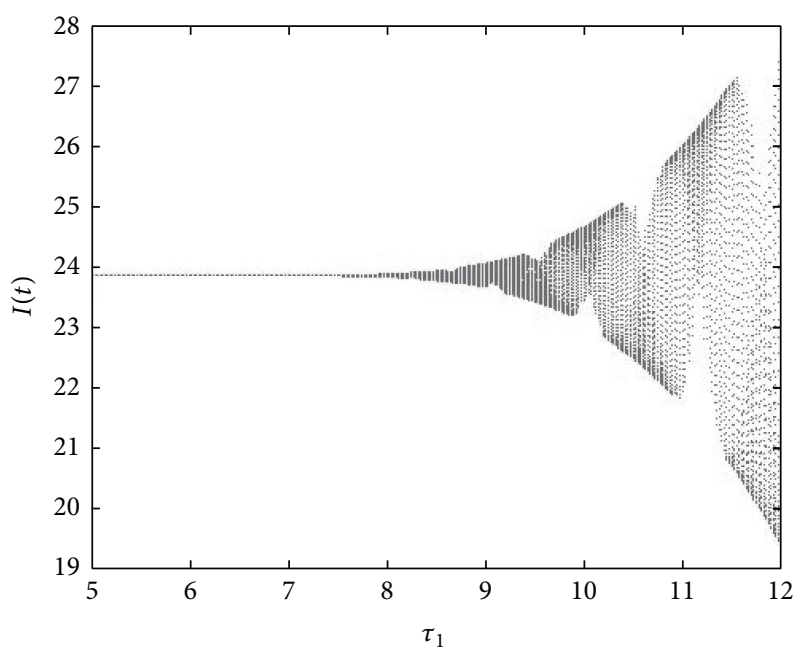

FIGURE 1: Bifurcation regarding $\tau_{1}$ with $\tau_{2}=0$.

$$
\begin{aligned}
& \frac{d R(t)}{d t}=0.4 I\left(t-\tau_{2}\right)-0.003 R(t)-0.3 R\left(t-\tau_{2}\right) \\
& \frac{d V(t)}{d t}=0.32 S(t)-0.003 V(t)-0.06 V\left(t-\tau_{2}\right) .
\end{aligned}
$$

It is easy to get $R_{0}=4.8750>1$ and system (72) has a unique positive equilibrium $D_{*}(1.5956,44.7772,23.6666$, $31.2430,8.1046)$. Also we get that the condition $\left(H_{1}\right)$ holds.

Firstly, we can obtain $\omega_{10}=0.2162$ and $\tau_{10}=$ 7.9833 by some computations. According to Theorem 1 , we can conclude that the positive equilibrium $D_{*}(1.5956$, $44.7772,23.6666,31.2430,8.1046)$ is asymptotically stable for $\tau_{1}<\tau_{10}=7.9833$. In this case, the propagation of worms can be predicted and controlled easily. However, once the value of $\tau_{1}$ is above $\tau_{10}=7.9833$, a Hopf bifurcation will occur which implies that the propagation of worms will be out of control. This property can be illustrated by the bifurcation diagram with respect to $\tau_{1}$ in Figure 1. Similarly, we have $\omega_{20}=0.4883$ and $\tau_{20}=2.4273$. The corresponding bifurcation diagram with respect to $\tau_{2}$ is as shown in Figure 2 .

Secondly, we may obtain $\omega_{10}^{*}=1.8164$ and $\tau_{10}^{*}=3.5477$ for $\tau_{1}>0$ and $\tau_{2}=1.5 \in\left(0, \tau_{20}\right)$ by computing. It follows from Theorem 3 that when $\tau_{1}=\tau_{10}^{*}=3.5477$, a Hopf bifurcation occurs, which can be shown by the bifurcation diagram regarding $\tau_{1}$ and $\tau_{2}=1.5$ in Figure 3. Moreover, we have $C_{1}(0)=-1.2298+3.8550 i, \mu_{2}=455.4815>0, \beta_{2}=$ $-2.4596<0$, and $T_{2}=-22.5006<0$. Thus, It follows from Theorem 5 that the Hopf bifurcation is supercritical and the bifurcating periodic solutions are stable and decreasing. Since the bifurcating periodic solutions are stable, the numbers of every class of nodes in system (72) may coexist in an oscillatory mode, which is not welcome in networks.

Lastly, by computing, we obtain $\omega_{20}^{*}=2.0979$ and $\tau_{20}^{*}=$ 1.5536 for $\tau_{2}>0$ and $\tau_{1}=3.5 \in\left(0, \tau_{10}\right)$. By Theorem 4, we know that the positive equilibrium $D_{*}(1.5956,44.7772$, $23.6666,31.2430,8.1046)$ is asymptotically stable for $\tau_{2}<$ $\tau_{20}^{*}$, which makes it possible for the propagation of worms

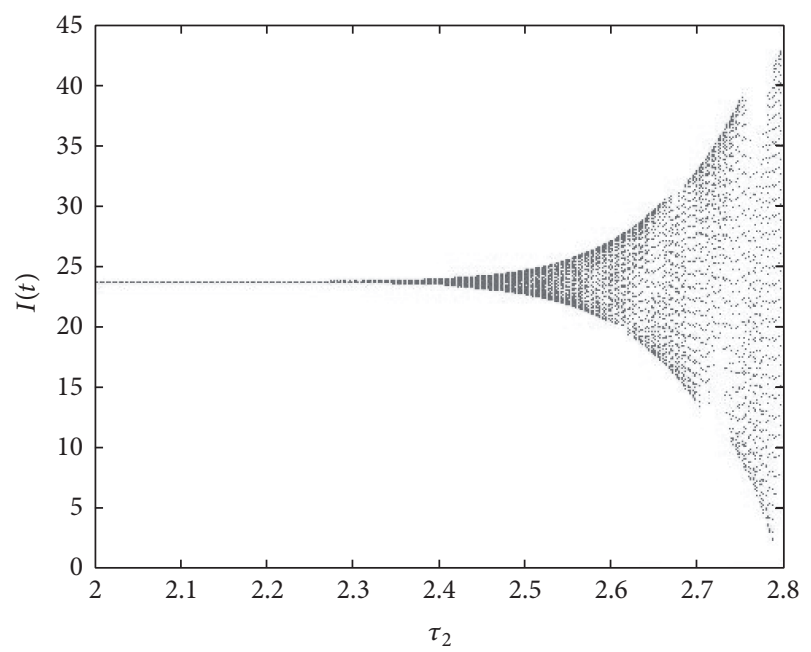

FIGURE 2: Bifurcation regarding $\tau_{2}$ with $\tau_{1}=0$.

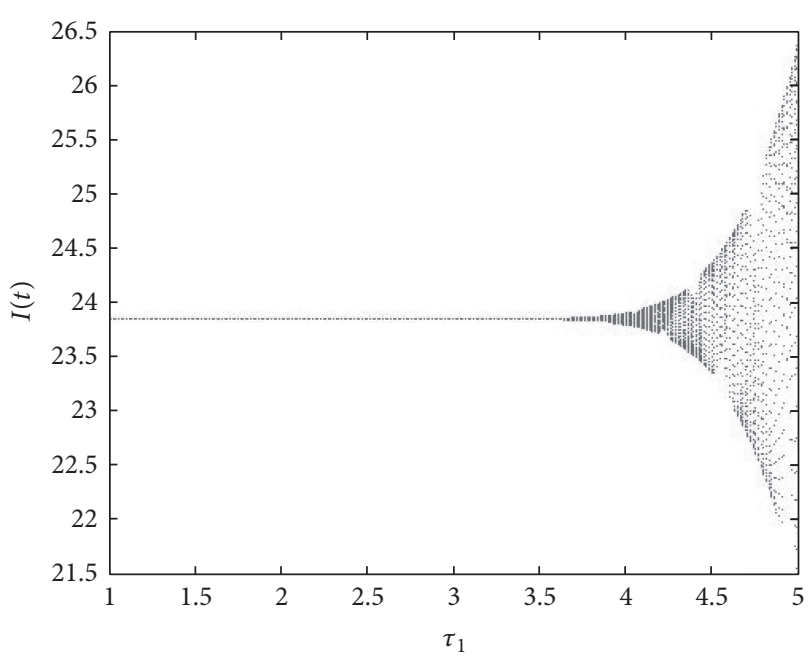

FIGURE 3: Bifurcation regarding $\tau_{1}$ with $\tau_{2}=1.5$.

to be predicted and controlled by taking effective measures. However, the positive equilibrium $D_{*}(1.5956,44.7772$, 23.6666, 31.2430, 8.1046) loses stability and a Hopf bifurcation occurs, when $\tau_{2}$ passes through $\tau_{20}^{*}=1.5536$. In this case, it is difficult to take measures to control the propagation of worms. The Hopf bifurcation phenomenon can be illustrated by Figure 4 .

\section{Conclusions}

In the present paper, the generalization of the delayed SEIRS$\mathrm{V}$ model describing worms spreading in a wireless sensor network investigated in [11] by inserting the latent period delay in the exposed sensor nodes has been considered. We find that not only $\tau_{1}$ but also $\tau_{2}$ can influence stability of system (4) and make system (4) undergo a Hopf bifurcation under some certain conditions. It has been shown that characteristics of the propagation of worms in system (4) can be easily predicted and eliminated when the value of delay is 


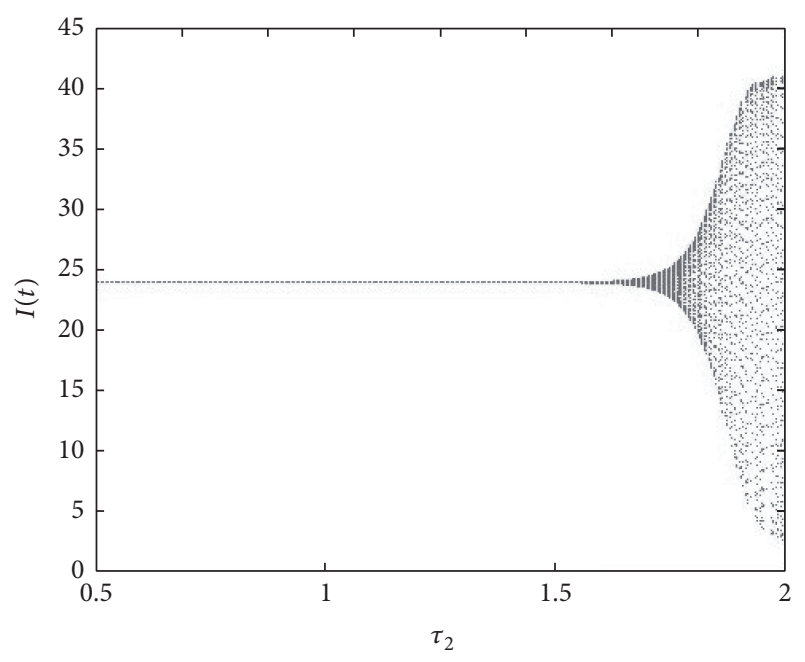

FIGURE 4: Bifurcation regarding $\tau_{2}$ with $\tau_{1}=3.5$.

below the corresponding critical value and the propagation of worms in system (4) may be out of control once the delay passes through the corresponding critical value. Thus, we can conclude that the worms propagation can be controlled by postponing occurrence of a Hopf bifurcation. Subsequently, we use the normal form approach theory and center manifold theory introduced in [17] to deal with properties of the Hopf bifurcation when $\tau_{1}>0$ and $\tau_{2} \in\left(0, \tau_{20}\right)$.

According to the numerical example, we can conclude that the dynamics of system (4) is more complicated than that of the system considered in [11]. However, it should be pointed out that we assume that the exposed sensor nodes can not infect other nodes, which is not consistent with reality, because the exposed nodes can also infect other nodes through vulnerability seeking or other methods. Consequently, it is more realistic to investigate dynamics of the proposed model in this paper with graded infection rate. We leave this as our future work.

\section{Competing Interests}

The authors declare that there is no conflict of interests regarding the publication of this paper.

\section{Acknowledgments}

This research was supported by Natural Science Foundation of Anhui Province (nos. 1608085QF145 and 1608085QF151) and Natural Science Foundation of the Higher Education Institutions of Anhui Province (no. KJ2015A144).

\section{References}

[1] T. Dargahi, H. H. S. Javadi, and M. Hosseinzadeh, "Applicationspecific hybrid symmetric design of key pre-distribution for wireless sensor networks," Security and Communication Networks, vol. 8, no. 8, pp. 1561-1574, 2015.

[2] M. E. J. Newman, "Spread of epidemic disease on networks," Physical Review E, vol. 66, no. 1, Article ID 016128, 11 pages, 2002.
[3] S. A. Khayam and H. A. Radha, "A topologically-aware worm propagation model for wireless sensor networks," in Proceedings of the 25th IEEE International Conference on Distributed Computing Systems Workshops (ICDCSW '05), pp. 210-216, Columbus, Ohio, USA, June 2005.

[4] K. T. Siok and A. Munro, "Adaptive probabilistic epidemie protocol for wireless sensor networks in an urban environment," in Proceedings of the 16th International Conference on Computer Communications and Networks (ICCCN '07), pp. 1105-1110, Honolulu, Hawaii, USA, August 2007.

[5] S. Tang and B. L. Mark, "Analysis of virus spread in wireless sensor networks: an epidemic model," in Proceedings of the 7th International Workshop on the Design of Reliable Communication Networks (DRCN '09), pp. 86-91, October 2009.

[6] S. S. Tang, "A modified SI epidemic model for combating virus spread in wireless sensor networks," International Journal of Wireless Information Networks, vol. 18, no. 4, pp. 319-326, 2011.

[7] W. H. Murray, "The application of epidemiology to computer viruses," Computers and Security, vol. 7, no. 2, pp. 139-145, 1988.

[8] J. O. Kephart and S. R. White, "Directed-graph epidemiological models of computer viruses," in Proceedings of the IEEE Computer Society Symposium on Research in Security and Privacy, pp. 343-359, IEEE, Oakland, Calif, USA, May 1991.

[9] J. O. Kephart and S. R. White, "Measuring and modeling computer virus prevalence," in Proceedings of IEEE Computer Society Symposium on Research in Security and Privacy, pp. 2-15, May 1993.

[10] B. K. Mishra and N. Keshri, "Mathematical model on the transmission of worms in wireless sensor network," Applied Mathematical Modelling, vol. 37, no. 6, pp. 4103-4111, 2013.

[11] Z. Z. Zhang and F. S. Si, "Dynamics of a delayed SEIRS-V model on the transmission of worms in a wireless sensor network," Advances in Difference Equations, vol. 2014, no. 1, article 295, 2014.

[12] C. Xu, X. Tang, and M. Liao, "Stability and bifurcation analysis on a ring of five neurons with discrete delays," Journal of Dynamical and Control Systems, vol. 19, no. 2, pp. 237-275, 2013.

[13] L. Gori, L. Guerrini, and M. Sodini, "Hopf bifurcation in a cobweb model with discrete time delays," Discrete Dynamics in Nature and Society, vol. 2014, Article ID 137090, 8 pages, 2014.

[14] C. Bianca, M. Ferrara, and L. Guerrini, "The time delays' effects on the qualitative behavior of an economic growth model," Abstract and Applied Analysis, vol. 2013, Article ID 901014, 10 pages, 2013.

[15] X.-Y. Meng, H.-F. Huo, X.-B. Zhang, and H. Xiang, "Stability and Hopf bifurcation in a three-species system with feedback delays,” Nonlinear Dynamics, vol. 64, no. 4, pp. 349-364, 2011.

[16] T. Zhang, H. Jiang, and Z. Teng, "On the distribution of the roots of a fifth degree exponential polynomial with application to a delayed neural network model," Neurocomputing, vol. 72, no. 46, pp. 1098-1104, 2009.

[17] B. D. Hassard, N. D. Kazarinoff, and Y. H. Wan, Theory and Applications of Hopf Bifurcation, Cambridge University Press, Cambridge, UK, 1981.

[18] C. Bianca, M. Ferrara, and L. Guerrini, "Qualitative analysis of a retarded mathematical framework with applications to living systems," Abstract and Applied Analysis, vol. 2013, Article ID 736058, 7 pages, 2013.

[19] T. K. Kar and A. Ghorai, "Dynamic behaviour of a delayed predator-prey model with harvesting," Applied Mathematics and Computation, vol. 217, no. 22, pp. 9085-9104, 2011. 
[20] C. Bianca and L. Guerrini, "Existence of limit cycles in the Solow model with delayed-logistic population growth," The Scientific World Journal, vol. 2014, Article ID 207806, 8 pages, 2014.

[21] M. Ferrara, L. Guerrini, and G. M. Bisci, "Center manifold reduction and perturbation method in a delayed model with a mound-shaped cobb-douglas production function," Abstract and Applied Analysis, vol. 2013, Article ID 738460, 6 pages, 2013.

[22] X.-Y. Meng, H.-F. Huo, and H. Xiang, "Hopf bifurcation in a three-species system with delays," Journal of Applied Mathematics and Computing, vol. 35, no. 1-2, pp. 635-661, 2011. 


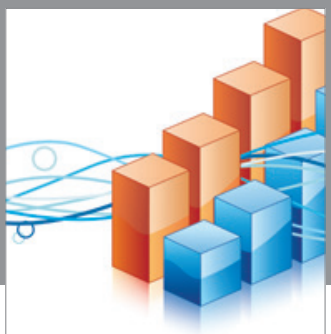

Advances in

Operations Research

vatem alat4

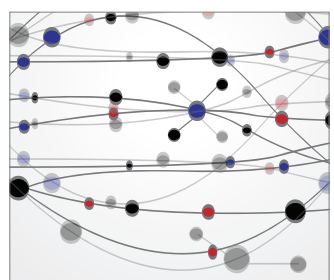

\section{The Scientific} World Journal
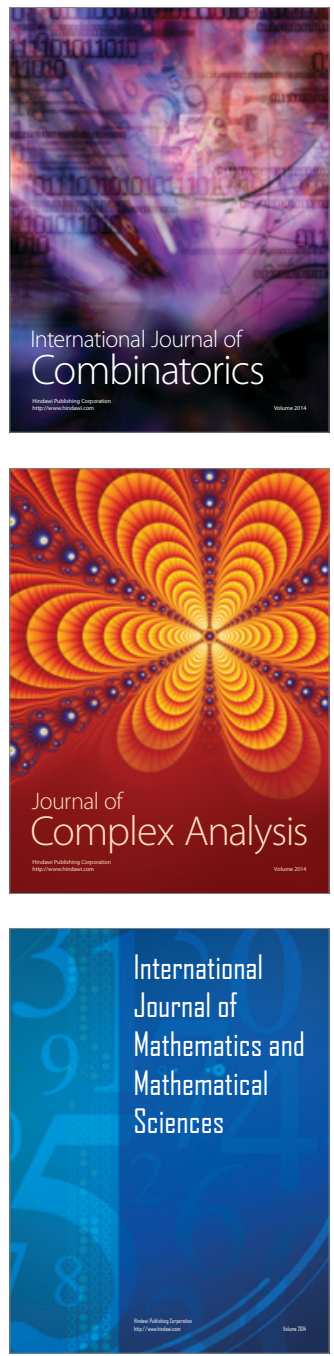
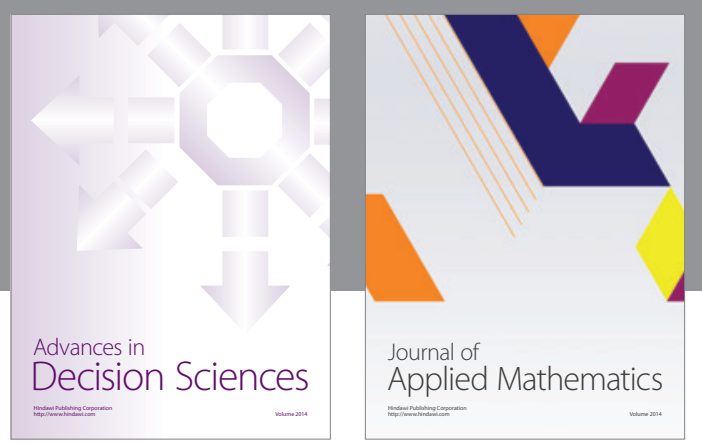

Algebra

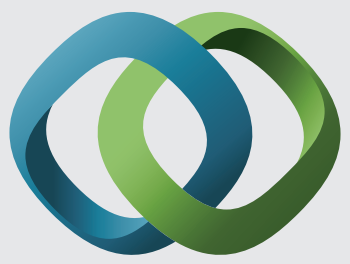

\section{Hindawi}

Submit your manuscripts at

https://www.hindawi.com
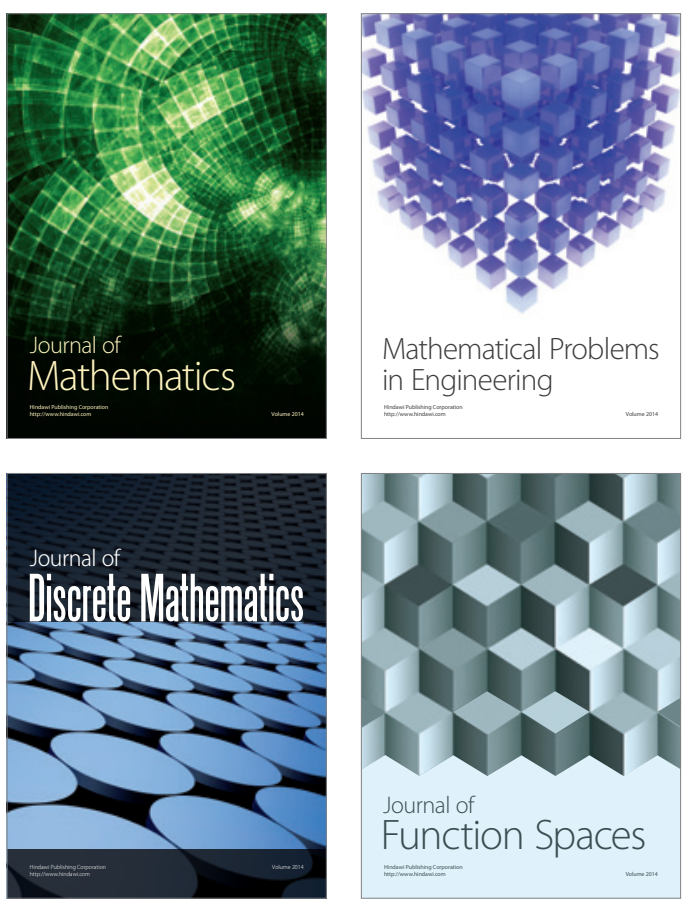

Mathematical Problems in Engineering
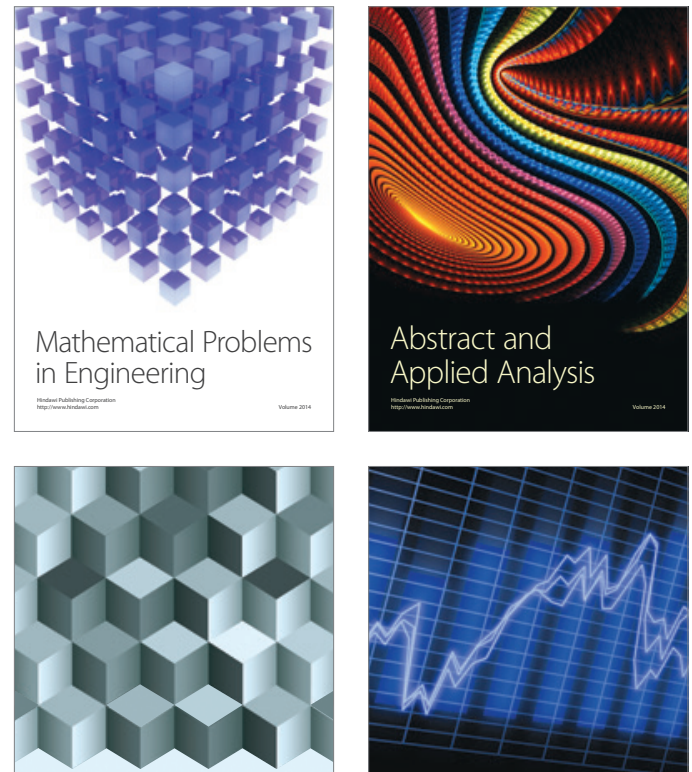

Journal of

Function Spaces

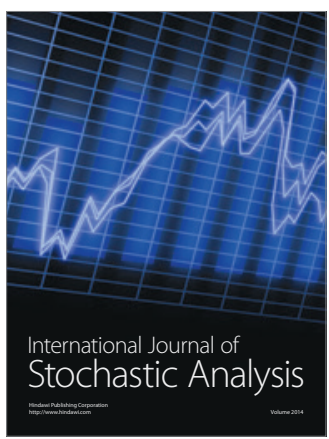

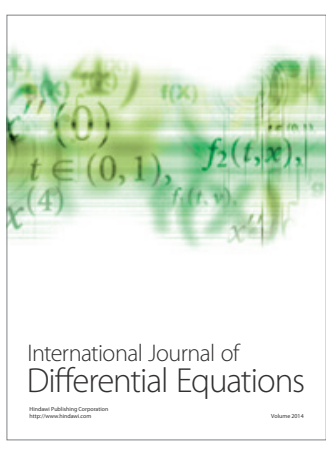
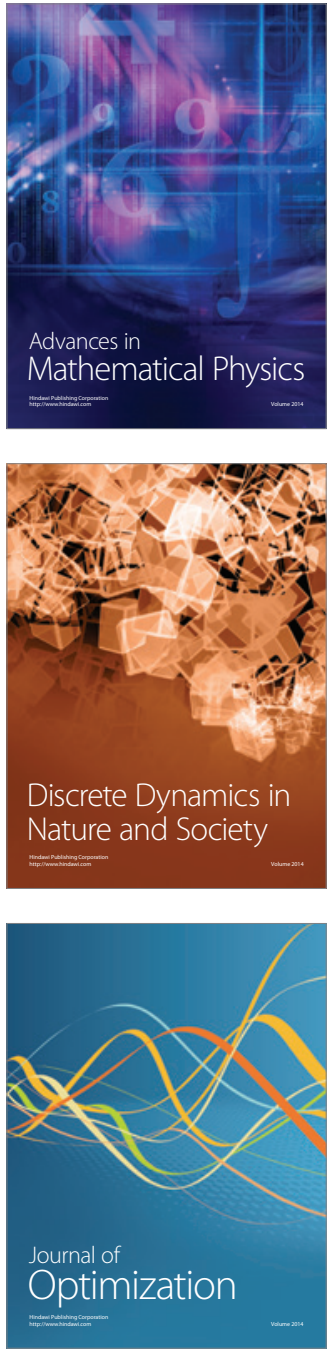